\title{
Diferencias en paisajes sonoros de sistemas silvopastoriles y potreros tradicionales del piedemonte llanero, Meta, Colombia
} Differences in soundscapes of silvopastoral systems and
traditional paddocks of the piedmont plain, Meta, Colombia

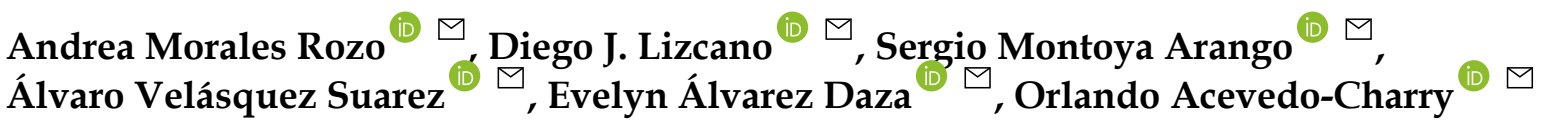

\section{Resumen}

Registramos el paisaje sonoro en cuatro fincas ganaderas en el piedemonte llanero del departamento del Meta, Colombia. Se utilizaron ocho grabadoras acústicas, cuatro en silvopastoriles de potreros arbolados y cuatro en sistemas de ganadería tradicional. Cada grabadora estuvo grabando 1 minuto cada 10 minutos, durante un promedio de 4 días, en noviembre de 2019. Se obtuvieron 4743 grabaciones de un minuto, $57.3 \%$ para sistemas de potreros tradicionales y $42.7 \%$ para potreros arbolados. Se encontraron diferencias en la actividad acústica y el uso del espacio acústico, el cual está relacionado a la distancia a las quebradas. Las variables distancia a vías y a quebradas explicaron la variación de riqueza de aves y anuros, la cual fue menor para potreros arbolados en relación a los potreros tradicionales, lo que puede estar relacionado con el diseño del sistema silvopatoril (monocultivo de falso yopo, Mimosa trianae). Futuras iniciativas silvopastoriles deberían incluir diseños más diversos para poder contrastar el tipo de métricas evaluadas en este estudio.

Palabras clave. Anuros. Aves. Colombia. Detecciones. Espacio acústico. Huella acústica. Orinoquia.

\begin{abstract}
We recorded the soundscape at four cattle ranches at the foothills of the Meta department, Colombia. We used eight acoustic recorders, four in silvopastoral systems and four in traditional cattle systems. Each recorder operated for 1 minute every 10 minutes, for an average of 4 days, in November 2019. We obtained 4743 one-minute recordings, $57.3 \%$ for traditional cattle systems and $42.7 \%$ for silvopastoral systems. Differences were found in the acoustic activity and use of the acoustic space, which are related to the distance to the streams. The variables distance to roads and streams explain the variation in richness of bird and anuran, which was lower in silvopastoral systems than traditional cattle systems. This may be related to the design of the silvopatoral system (Mimosa trianae monoculture). Future silvopastoral initiatives should include more diverse designs in order to contrast the type of metrics evaluated in this study.
\end{abstract}

Key words: Acoustic space. Acoustic print. Anurans. Birds. Orinoco. 


\section{Introducción}

La ecología del paisaje sonoro es una ciencia emergente que permite tener una aproximación a la dinámica de los paisajes (Campos-Cerqueira \& Aide, 2017; Tucker et al., 2014) y ha venido desarrollando nuevas herramientas para su estudio (Depraetere et al., 2012; Pekin et al., 2012; Pijanowski et al., 2011; Sueur et al., 2008). Particularmente, la ecología de los paisajes sonoros usa el sonido para investigar los procesos que afectan la diversidad biológica de un ecosistema y recopilar información sobre la composición, estructura y actividad acústica de la fauna, y cómo se relaciona esto con ciertas variables de paisaje (Campos-Cerqueira \& Aide, 2017; Joo et al., 2011; Rodríguez-Buriticá et al., 2018; Tucker et al., 2014). El monitoreo acústico automatizado se ha vuelto más popular y asequible desde la aparición de equipos de grabación portátiles y de bajo costo (Gibb et al., 2019), los cuales permiten cuantificar la biodiversidad a través de la actividad vocal de una comunidad en un ecosistema y evidenciar cambios a través del espacio y el tiempo (Depraetere et al., 2012). Además, este método automatizado ha sido implementado en el estudio de ciertos grupos taxonómicos, entre los que se destacan los insectos (Manakin et al., 2011), anuros (Ospina et al., 2013), mamíferos (Kalan et al., 2015), aves (Campos-Cerqueira \& Aide, 2016), o a escala de comunidades (Aide et al., 2017).

Recientemente se estableció la Red de Ecoacústica Colombiana, que generó el primer reporte de paisajes sonoros para Colombia, con 330 áreas de estudio de monitoreo acústico pasivo. Estas áreas incluyen ecosistemas naturales poco perturbados y transformados (Rodríguez-Buriticá et al., 2018). Sin embargo, para la Orinoquia colombiana no existen estudios de este tipo que permitan describir la biodiversidad desde una dimensión acústica (Rodríguez-Buriticá et al., 2018). En esta región, los procesos de ganadería extensiva y el desarrollo agroindustrial han generado cambios en los usos del suelo (Romero-Ruiz et al., 2012), lo cual se ha acelerado en el nuevo escenario de postacuerdo, sumado a la alta presión de desarrollo agroindustrial (Williams et al., 2020). Esta transformación es evidente especialmente en el departamento del Meta, donde la tasa de deforestación en 2018 fue una de las más altas en el país, principalmente asociada a actividades agrícolas a gran escala, como ganadería extensiva y cultivos ilícitos (IDEAM, 2019). Bajo este desalentador escenario, una de las estrategias de conservación que se ha venido impulsando a través del proyecto Ganadería
Colombiana Sostenible es la implementación de sistemas silvopastoriles como sistemas productivos que promueven la diversidad biológica en zonas ganaderas y agrícolas homogéneas (Murgueitio et al., 2013), al generar pequeñas manchas aisladas de vegetación y corredores de hábitat heterogéneos (Naranjo, 1992).

Los sistemas silvopastoriles son un escenario interesante para estudiar cómo la heterogeneidad espacial influye en la estructura y el funcionamiento de los ecosistemas $(\mathrm{Wu}, 2004)$. Esta heterogeneidad generada por los sistemas silvopastoriles puede verse reflejada en variaciones de composición del paisaje acústico (Bormpoudakis et al., 2013). A pesar de que son pocos los estudios de biodiversidad en sistemas silvopastoriles en Colombia, se han encontrado diferencias en la composición y estructura de las comunidades de aves entre sistemas silvopastoriles y sistemas productivos no silvopastoriles (Fajardo et al., 2009). Teniendo en cuenta que las comunidades de aves pueden ser diferentes y que las comunicaciones acústicas de los animales se reflejan directamente en el paisaje sonoro (Aide et al., 2017), esperamos encontrar diferencias entre los paisajes sonoros de sistemas silvopastoriles y los sistemas de ganadería tradicional.

Con base en lo anterior, el objetivo general de este estudio fue evaluar si existen diferencias entre el uso del espacio acústico en sistemas silvopastoriles y sistemas ganaderos tradicionales en el piedemonte llanero de la Orinoquia colombiana. En particular, analizamos los componentes del paisaje sonoro, a través de detecciones de dos grupos indicadores y mejor conocidos en su acústica (aves y anuros) y diferencias entre el número de especies acústicamente activas en estos sistemas productivos.

\section{Materiales y métodos}

Área de estudio. El trabajo se desarrolló en el piedemonte llanero de la Orinoquia colombiana en el departamento del Meta (Figura 1). El muestreo se realizó en cuatro fincas que hacen parte del proyecto Ganadería Colombiana Sostenible (http://ganaderiacolombianasostenible.co), con elevaciones entre 424 y 738 m s.n.m: El Porvenir, con 37 ha $y$ 70-80 cabezas de ganado (Dorado); La Rosania, con 79 ha y 150 cabezas de ganado; la finca Andorra, con 257 ha y 421 cabezas de ganado; y finca La Pradera con 217 ha y 316 cabezas de ganado (San Luis Cubarral; Tabla 1). 
La zona de piedemonte del Meta tiene una humedad que oscila entre el $47 \%$ y el $75 \%$, temperatura promedio de $26^{\circ} \mathrm{C}$ y una precipitación anual unimodal de 4500 $\mathrm{mm}$, con una temporada de lluvias de abril a noviembre (IDEAM, 2005). Los sistemas de ganadería tradicional usan potreros tradicionales sin árboles y dominados por pastos exóticos, como Brachiaria spp. Aunque existen diferentes sistemas silvopastoriles en el piedemonte, en este estudio se incluyeron solo potreros con árboles dispersos o potreros arbolados con falso yopo (Mimosa trianae), una especie arbórea nativa con un dosel poco denso que permite la filtración de la luz; además, sus foliolos pequeños se descomponen rápidamente, retienen agua y son claves en la fijación de nitrógeno (Calle et al., 2012). Los árboles en los potreros tenían entre 6 y 12 años al momento del estudio. La finca El Porvenir era la única donde los potreros tradicionales estaban junto a una plantación de Theobroma cacao de 6 a 8 años. Los bosques nativos dentro de las fincas estaban asociados a cursos de agua, y se caracterizaban por tener árboles de 12-25 m. Adicionalmente, todas las fincas utilizan cercas vivas para dividir potreros o predios.

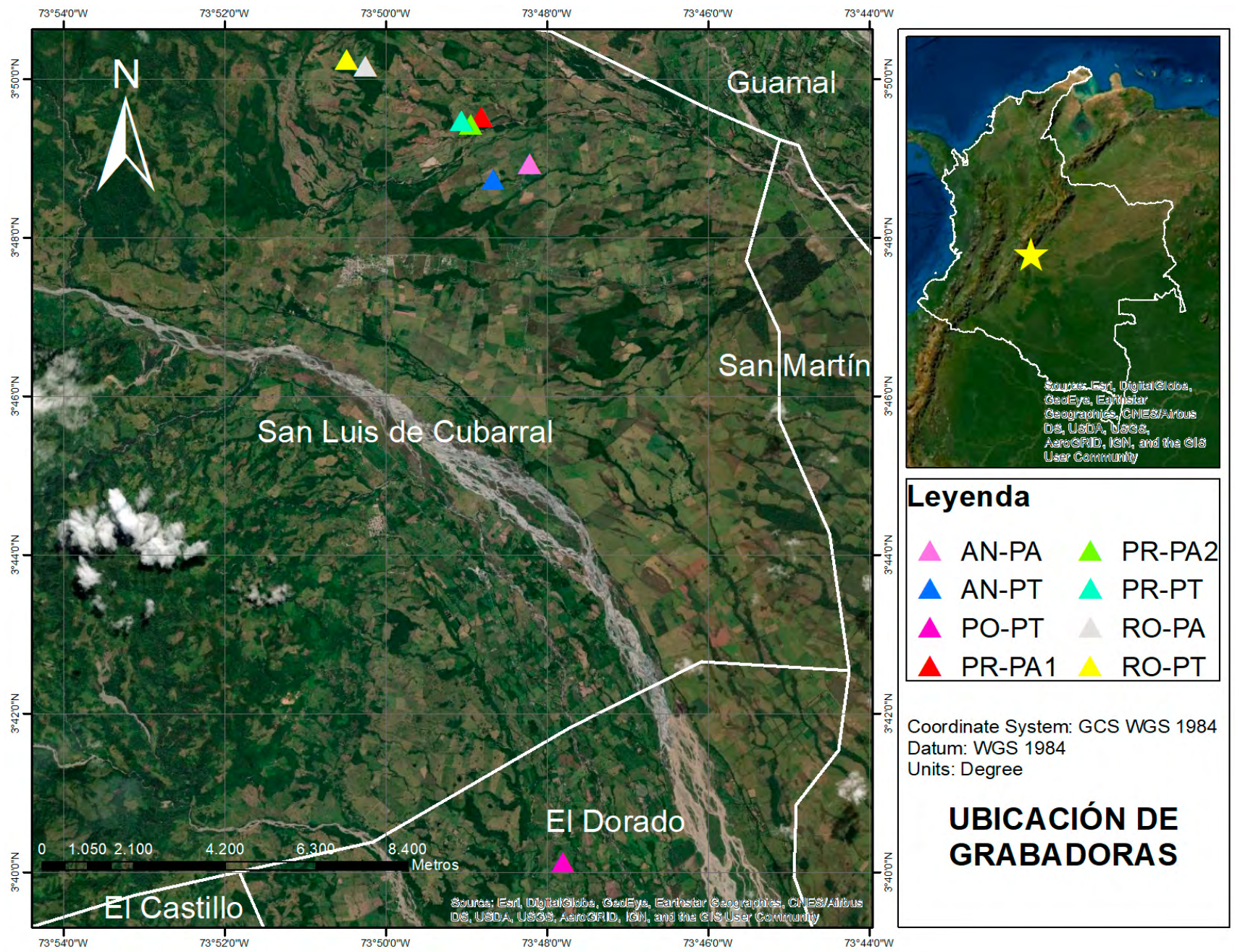

Figura 1. Ubicación de las fincas donde se muestrearon los paisajes sonoros en el departamento del Meta, Colombia. AN, Andorra; PO, El Porvenir; PR La Pradera; RO, La Rosanía; PA, potreros arbolados (sistemas silvopastoriles); PT potreros tradicionales.

Figure 1. Location of the farms where the soundscapes were sampled in the department of Meta, Colombia. AN, Andorra; PO, El Porvenir; PR La Pradera; RO, La Rosanía; PA, wooded pastures (silvopastoral systems); PT traditional paddocks. 
Tabla 1. Características de los sitios donde se muestrearon los paisajes sonoros en el departamento del Meta, Colombia. PA, potreros arbolados (sistemas silvopastoriles); PT, potreros tradicionales.

Table 1. Characteristics of the sites where soundscapes were studied in the department of Meta, Colombia. PA, wooded pasture (silvopastoral systems); PT, traditional pasture.

\begin{tabular}{cccccccc}
\hline Finca & Sistema & Nombre sitio & Latitud & Longitud & $\begin{array}{c}\text { Elevación } \\
(\mathbf{m})\end{array}$ & Grabaciones & Días \\
\hline Porvenir & PT & PT_Porvenir & $3^{\circ} 40^{\prime} 07.39^{\prime \prime} \mathrm{N}$ & $73^{\circ} 22^{\prime} 46.83^{\prime \prime} \mathrm{O}$ & 424 & 719 & 4.99 \\
\hline Andorra & PT & PT_Andorra & $3^{\circ} 48^{\prime} 44.50^{\prime \prime} \mathrm{N}$ & $7^{\circ} 48^{\prime} 41.04^{\prime \prime} \mathrm{O}$ & 534 & 727 & 5.05 \\
\hline Pradera & PT & PT_Pradera & $3^{\circ} 49^{\prime} 28.78^{\prime \prime} \mathrm{N}$ & $7^{\circ} 49^{\prime} 04.04^{\prime \prime} \mathrm{O}$ & 560 & 541 & 3.76 \\
\hline Rosania & PT & PT_Rosania & $3^{\circ} 50^{\prime} 15.04^{\prime \prime} \mathrm{N}$ & $7^{\circ} 50^{\prime} 29.29^{\prime \prime} \mathrm{O}$ & 770 & 730 & 5.07 \\
\hline Andorra & PA & PA_Andorra & $3^{\circ} 48^{\prime} 56.23^{\prime \prime} \mathrm{N}$ & $7^{\circ} 48^{\prime} 13.07^{\prime \prime} \mathrm{O}$ & 520 & 728 & 5.06 \\
\hline Pradera & PA & PA_1Pradera & $3^{\circ} 49^{\prime} 31.66^{\prime \prime} \mathrm{N}$ & $7^{\circ} 48^{\prime} 49.36^{\prime \prime} \mathrm{O}$ & 525 & 437 & 3.03 \\
\hline Pradera & PA & PA_2Pradera & $3^{\circ} 49^{\prime} 26.00^{\prime \prime} \mathrm{N}$ & $7^{\circ} 48^{\prime} 56.74^{\prime \prime} \mathrm{O}$ & 525 & 142 & 0.99 \\
\hline Rosania & PA & PA_Rosania & $3^{\circ} 50^{\prime} 09.74^{\prime \prime} \mathrm{N}$ & $7^{\circ} 50^{\prime} 15.11^{\prime \prime} \mathrm{O}$ & 738 & 729 & 5.06 \\
\hline
\end{tabular}

Toma de datos. Entre el 9 y el 27 de noviembre de 2019 se instalaron grabadoras en sistemas silvopastoriles de árboles dispersos de falso yopo ( $M$. trianae) y en sistemas de ganadería tradicional. Usamos sistemas de grabación pasiva ARBIMON de Sieve Analytics, por medio de la aplicación ARBIMON Touch para Android, instalada en teléfonos celulares marca Motorola MotoG1, los cuales se instalaron dentro de una caja plástica (Grace Digital Eco Pod) que protegía el dispositivo de la lluvia. Cada celular se conectó a un micrófono (Monoprice) que permitía ampliar el rango de grabación de los teléfonos celulares (Herrera-Montes, 2018). Los teléfonos se programaron con la aplicación para grabar a una frecuencia de $44.1 \mathrm{kHz}$ con 16 bits de resolución durante 1 minuto, a intervalos de 10 minutos por un promedio de 4 días en cada lugar (Tabla 1), generando grabaciones con formato no comprimido WAV. Las grabadoras se instalaron manteniendo una distancia mínima de 200 metros, a una altura de 1.50 metros del suelo y aseguradas con cuerdas a un tronco de árbol, con el micrófono dirigido hacia abajo (Herrera-Montes, 2018).

Covariables ambientales. Para evaluar el efecto en el paisaje sonoro, se definieron covariables ambientales a partir de la imágen del satélite Sentinel-2 del 25 de diciembre de 2018 (Dymond et al., 2002; Samarawickrama et al., 2017), que permite una resolución entre 10 y $20 \mathrm{~m}$ (Vrieling et al., 2018). Se calculó el porcentaje de bosque natural (incluso secundario), potrero arbolado y potrero tradicional en radios de 50, 100 y $200 \mathrm{~m}$
(Información Suplementaria y Tabla S1). Aunque las variables de porcentaje de bosque natural, potrero arbolado y potrero tradicional a diferentes radios estuvieron altamente correlacionadas, para las comparaciones se usaron solo los valores a un radio de $200 \mathrm{~m}$. Así mismo, se estimaron las distancias a vías y quebradas. Estas covariables ambientales fueron usadas para una escogencia múltiple de modelos lineales generalizados que pudieran explicar las variables respuesta, para lo cual fueron estandarizadas (i.e., reescalonadas para tener como promedio 0). Se usó la función glmulti del paquete glmulti para generar automáticamente todos los posibles modelos. Para determinar el modelo más factible o mejor ajustado se usó el criterio de información de Akaike (AICc), considerando un $\triangle \mathrm{AICc}<2$ para los modelos más robustos (Burnham \& Anderson, 2004).

Análisis de paisajes sonoros. Cada grabación fue normalizada a $-3 \mathrm{~dB}$ y se aplicó un filtro de paso alto de $150 \mathrm{~Hz}$ en el programa Ocenaudio, con el fin de disminuir un poco la cantidad de viento en algunas grabaciones, que dificultaba la visualización de emisiones acústicas de aves y anuros. Posteriormente, se cargaron en la plataforma de análisis bioacústico ARBIMON 11 (https://arbimon.sieve-analytics.com) y se realizó un análisis de paisaje sonoro por agregación de grabaciones por hora, para obtener el comportamiento de la huella acústica de cada sitio (Campos-Cerqueira et al., 2019; Furumo \& Aide, 2019; Herrera-Montes, 2018). Para obtener los paisajes sonoros, se definió la agregación de 
las grabaciones en escala de tiempo de la hora del día (24 h), un tamaño de intervalo de frecuencia de $21 \mathrm{~Hz}$, un umbral de amplitud de 0.05 (relativo al pico máximo), y la distancia mínima entre picos a $0 \mathrm{~Hz}$. Los parámetros fueron escogidos primero siguiendo estudios publicados (Campos-Cerqueira et al., 2019; Furumo \& Aide, 2019; Herrera-Montes, 2018), y luego manteniendo la mejor representación ajustada a nuestros datos durante un análisis preliminar. La configuración de análisis de paisajes sonoros genera un total de 25200 celdas ( 24 horas x 1050 bandas de frecuencia) para cada sitio, con información en cada celda de la proporción de grabaciones con picos de actividad acústica por encima del umbral definido.

Para comparar los paisajes sonoros, se realizó una ordenación no métrica multidimensional escalonada (NMDS) a partir de las celdas que arrojaron más información en alguno de los sitios (sumatoria de proporciones de grabaciones entre los sitios $>1$ ). Dichas celdas (806) incluyeron actividad acústica concentrada principalmente en frecuencias del espectro audible por debajo de los $10 \mathrm{kHz}$, rango de frecuencia que incluye la mayoría de los sonidos de aves y anfibios (Aide et al., 2017); por eso centramos los análisis para la ordenación solo en esa franja de frecuencia audible $(0-10 \mathrm{kHz})$. La ordenación se corrió usando la distancia de disimilitud Jaccard, al ser datos de incidencia, con 20 permutaciones y a partir del uso de la función metaMDS. La matriz usada para correr la ordenación también sirvió para comparar los paisajes sonoros y relacionarlos con diferentes covariables ambientales, a partir de un análisis de varianza (ANDEVA) multivariado, con la función adonis, usando 999 permutaciones. Finalmente, comparamos la dispersión de los puntos de cada sistema con la función betadisper. Las tres funciones mencionadas pertenecen al paquete vegan en R (Oksanen et al., 2019).

Composición del paisaje sonoro y actividad acústica de aves y anuros. Se revisaron 15 grabaciones por hora, por día, por sitio aleatoriamente en las franjas de horas 06:00-07:00, 12:00-13:00, 18:00-19:00 y 00:00-01:00 con el fin de identificar los principales componentes del paisaje sonoro. En el caso de la finca La Pradera, tanto para potreros arbolados como para potrero tradicional, solamente se encontraron 6 archivos para el periodo de muestreo, al parecer debido a un fallo con la grabadora, así que revisamos el total de archivos en estos sitios (Tabla 2). Se anotó la presencia y ausencia de componentes del paisaje sonoro usando la herramienta en ARBIMON II "Composición de paisaje sonoro"; se seleccionaron, para cada grabación revisada, presencia de geofonía (viento, lluvia, movimiento de agua), biofonía (aves, anfibios, insectos, mamíferos, domésticos) y antropofonía (vehículos terrestres, vehículos aéreos, maquinaria, voces, sonidos eléctricos).

Tabla 2. Número de archivos revisados para la composición del paisaje sonoro en el departamento del Meta, Colombia, para noviembre de 2019. PA, potrero arbolado; PT, potrero tradicional.

Table 2. Number of files revised for the composition of the soundscape in the department of Meta, Colombia, November 2019. PA, wooded pasture (silvopastoral systems); PT, traditional pasture.

\begin{tabular}{lcccccc}
\hline \multirow{2}{*}{ Sitio } & \multicolumn{5}{c}{ Hora } & \multirow{2}{*}{ Total } \\
\cline { 2 - 5 } & 12 AM & $\mathbf{6 ~ A M}$ & $\mathbf{1 2} \mathbf{P M}$ & $\mathbf{6 ~ P M}$ & \\
\hline PT_Porvenir_norm & 14 & 15 & 15 & 14 & 58 \\
\hline PT_Andorra_norm & 15 & 13 & 11 & 13 & 52 \\
\hline PT_Pradera_norm & 6 & 6 & 7 & 6 & 25 \\
\hline PT_Rosania_norm & 14 & 15 & 15 & 15 & 59 \\
\hline PA_Andorra_norm & 13 & 14 & 13 & 16 & 56 \\
\hline PA_1Pradera_norm & 14 & 11 & 15 & 15 & 55 \\
\hline PA_2Pradera_norm & 6 & 6 & 5 & 5 & 22 \\
\hline PA_Rosania_norm & 13 & 15 & 15 & 15 & 58 \\
\hline Total & $\mathbf{9 5}$ & $\mathbf{9 5}$ & $\mathbf{9 6}$ & $\mathbf{9 9}$ & $\mathbf{3 8 5}$ \\
\hline
\end{tabular}


Adicionalmente, se revisaron entre 30 y 40 grabaciones al azar de cada sitio durante el coro de amanecer (5:00-8:00) y el coro nocturno (17:00-20:00), identificando principalmente aves y anuros. Aunque los insectos son uno de los principales componentes de la biofonía en sistemas tropicales (Aide et al., 2017), centramos este análisis en aves y anuros, dado que la mayor concentración de actividad acústica fue por debajo de los $10 \mathrm{kHz}$, y para poder identificar fielmente la mayoría de fuentes de sonido biótico. La revisión significó otras 587 grabaciones diferentes a las anteriormente revisadas para este componente específico de la biofonía de cada paisaje sonoro. Para la identificación de las especies se usaron como referencias bases de datos de sonidos de aves como xeno-canto (https:/ / www.xeno-canto.org), Macaulay Library (https://www.macaulaylibrary.org), AmphibiaWeb (https:/ / amphibiaweb.org), batrachia (https:/ / www. batrachia.com) y archivos particulares de los autores. Con este conjunto de datos, comparamos las detecciones (número de grabaciones con presencia de alguna especie o componente del paisaje sonoro), la riqueza (número de especies del submuestreo) y composición (lista de especies) entre los sitios y tipos de potreros.

Para determinar las diferencias en la composición del paisaje sonoro entre los sistemas tradicionales y arbolados, se realizó un conteo de los dos grupos taxonómicos seleccionados (anuros y aves) y se estimó la riqueza de especies. Posteriormente, se realizó un modelo lineal generalizado que vinculaba la variable respuesta (conteo y riqueza de especies) con los factores fijos de sistema (tradicional o arbolado), componente (geofonía, biofonía, antropofonía) y grupo taxonómico (anuros y aves), así como sus interacciones. Cada modelo lineal generalizado fue vinculado con la función de enlace Poisson. Para determinar los tipos diferentes se realizó una prueba post-hoc de comparación múltiple de Tukey. Por su parte, se comparó la composición de especies entre los sitios con sistema tradicional o arbolado con el índice de disimilitud de Jaccard (Jaccard, 1912). Entre más altos los valores del índice de Jaccard, más alto el recambio o mayor disimilitud entre los sitios.

\section{Resultados}

Se obtuvieron 4743 grabaciones de un minuto, 2717 para sistemas de potreros tradicionales y 2026 para potreros arbolados (Tabla 1). En promedio se muestreo 4.99 días por sitio, con algunas excepciones en el PA de finca La Pradera (Tabla 1), debido a una falla en la grabadora. La huella acústica de cada sitio representó, en general, actividad acústica en horas de la noche y principalmente por debajo de los $7 \mathrm{kHz}$ de frecuencia (Figura 2A). El uso del espacio acústico en promedio fue del $4.46 \%$, pero varió entre sitios. Los sitios con mayor porcentaje de espacio acústico usado fueron PT_Andorra (20.04\%), PT_Pradera (16.16 \%) y PA_2Pradera $(14.18 \%)$. Por otro lado, los sitios con menor uso del espacio acústico fueron PT_Rosania (0.66 \%), PA_1Pradera (2.15\%), y PT_Porvenir (3.98 \%).

Al comparar los paisajes sonoros de los diferentes sitios por medio del NMDS (Figura 2B), el cual tuvo un valor de estrés de 0.048 con dos dimensiones (Figura S1), se observó que tanto los sistemas de potreros arbolados como los potreros tradicionales parecen distanciarse en el eje 2, con los potreros arbolados hacia valores positivos y potreros tradicionales hacia valores negativos, aunque la dispersión entre ellos no varió significativamente $\left(F_{1,6}=0.630, P=0.46\right)$. Aunque los sitios se ven aparentemente muy cercanos (promedio distancia 900 m, rango 241-15963 m), la distancia a la grabadora más cercana no se correlaciona con ninguno de los ejes del NMSD, es decir que la ordenación es independiente de la distribución espacial de los sitios. El análisis de varianza (ANDEVA) mostró una variación que estuvo significativamente relacionada con el espacio acústico usado (Figura $2 \mathrm{C} ; F_{1,7}=2.784, P=0.02$ ) y con la distancia a quebradas (Figura 2D; $F_{1,7}=2.092, P=0.05$ ).

Los componentes del paisaje sonoro variaron diferencialmente entre los dos tipos de sistemas de potreros (Figura 3A). Los potreros arbolados se caracterizaron por bajas detecciones de antropofonía, mientras que geofonía y biofonía fueron similares. En potreros tradicionales, las detecciones de geofonía fueron similares a antropofonía, mientras que las detecciones de biofonía fueron más altas que antropofonía. Todos los modelos mejor ajustados $(\triangle \mathrm{AICc}<2)$ para las detecciones de componentes de paisajes sonoros incluyeron la distancia a vías como variable más importante (Tabla S2, Figura S2).

La mayor diferencia de las detecciones del componente biofonía se encontró en las aves (Tabla 3). Las detecciones de aves fueron marginalmente diferentes entre los dos sistemas (Figura 3B, $P=0.08$ ). Sin embargo, se encontraron pocas detecciones de anuros en sistemas de potreros arbolados. Al igual que con las detecciones de componente de paisaje sonoro, todos los modelos mejor ajustados para las detecciones de los dos grupos taxonómicos revisados (aves y anuros) incluyeron la distancia a vías como variable más importante (Tabla S2, Figura S3). En cuanto al número de especies de anuros 
A
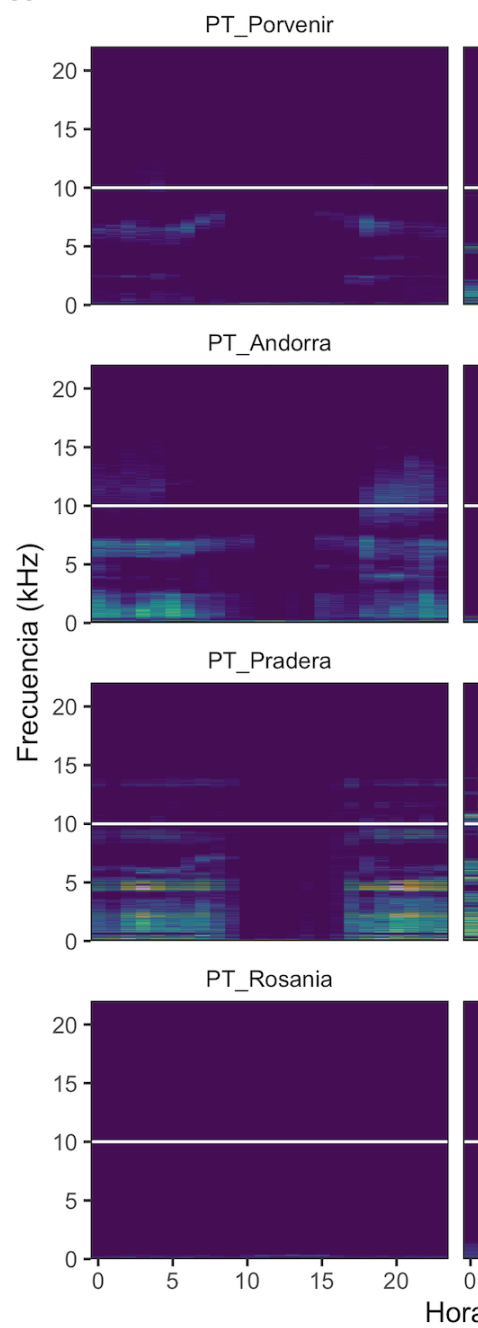

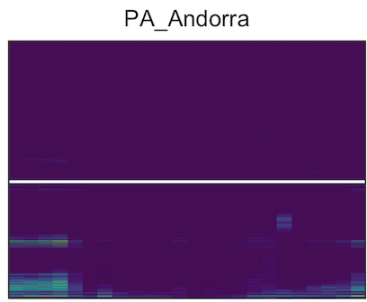

PA_1Pradera

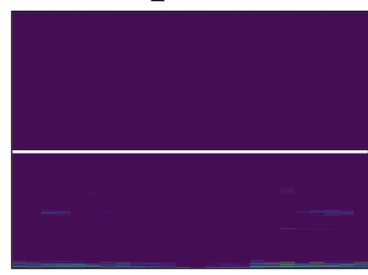

PA 2Pradera

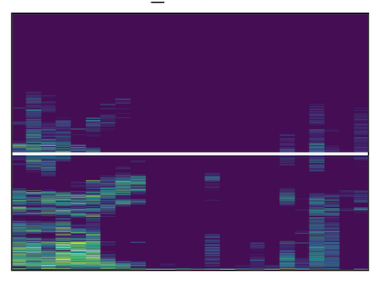

PA_Rosania

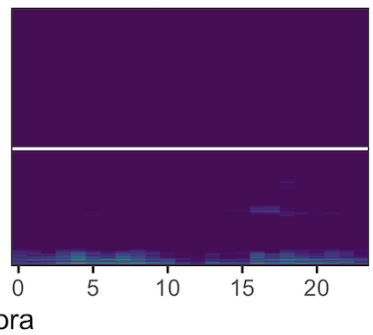

Z

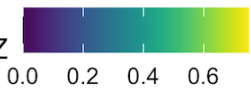

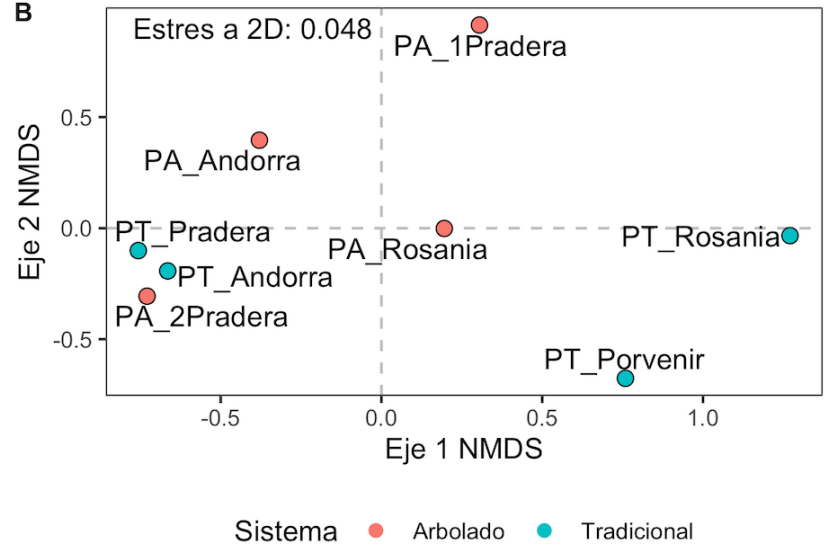

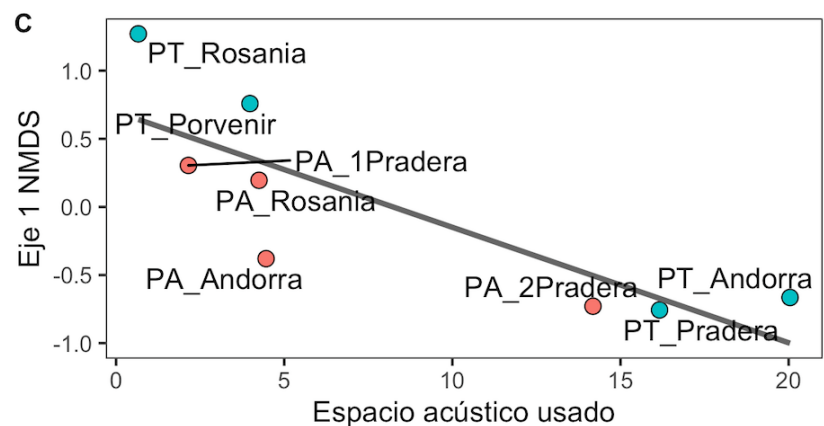

D

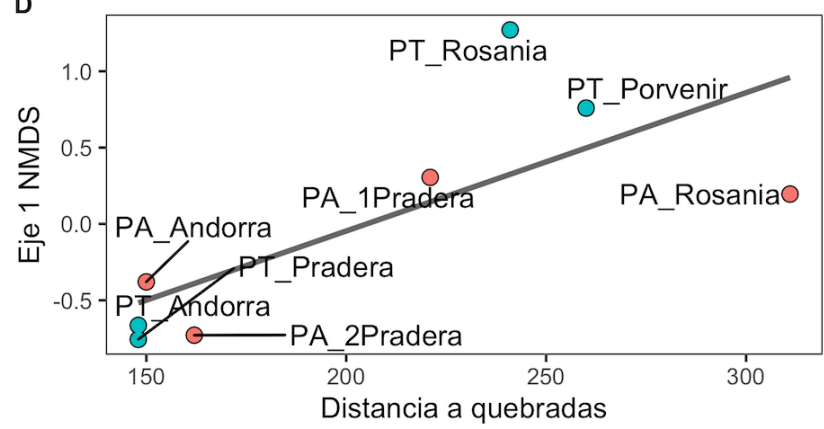

Figura 2. Representación visual (A) y comparación (B) del paisaje acústico de ocho sitios muestreados en el departamento del Meta, Colombia (ver Tabla 1 para descripción de los sitios). A, Los ejes representan la hora del día (X), frecuencia en $\mathrm{kHz}$ (Y) y proporción de grabaciones con actividad por encima de un umbral de 0.05 (Z); nótese que la mayoría de la actividad se concentra por debajo de los $10 \mathrm{kHz}$ (línea horizontal blanca). El uso del espacio se calculó por la suma de número de celdas de tiempo/banda de frecuencia que fuera ocupado. B, Ordenación multidimensional no métrica escalonada (NMDS) a partir de celdas de tiempo/banda de frecuencia por cada sitio con bandas de frecuencia hasta $10 \mathrm{kHz}$. Relación del eje 1 de NMDS con el espacio acústico usado (C) y con la distancia a quebradas (D).

Figure 2. Visual representation (A) and comparison (B) of the soundscape of eight sampled sites in the department of Meta, Colombia (see Table 1 for description of the sites). A, The axes represent the time of day (X), frequency in $\mathrm{kHz}(\mathrm{Y})$ and proportion of recordings with activity above a threshold of 0.05 (Z); note that almost all acoustic activity was concentrated below $10 \mathrm{kHz}$ (white horizontal line). The use of space was calculated by the sum of the number of time cells/frequency band that was occupied. B, Non-metric multidimensional scaling (NMDS) ordination from time cells/frequency band for each site with frequency bands up to $10 \mathrm{kHz}$. Relationship of the axis 1 of NMDS and acoustic space used (C) and distance to streams (D). 

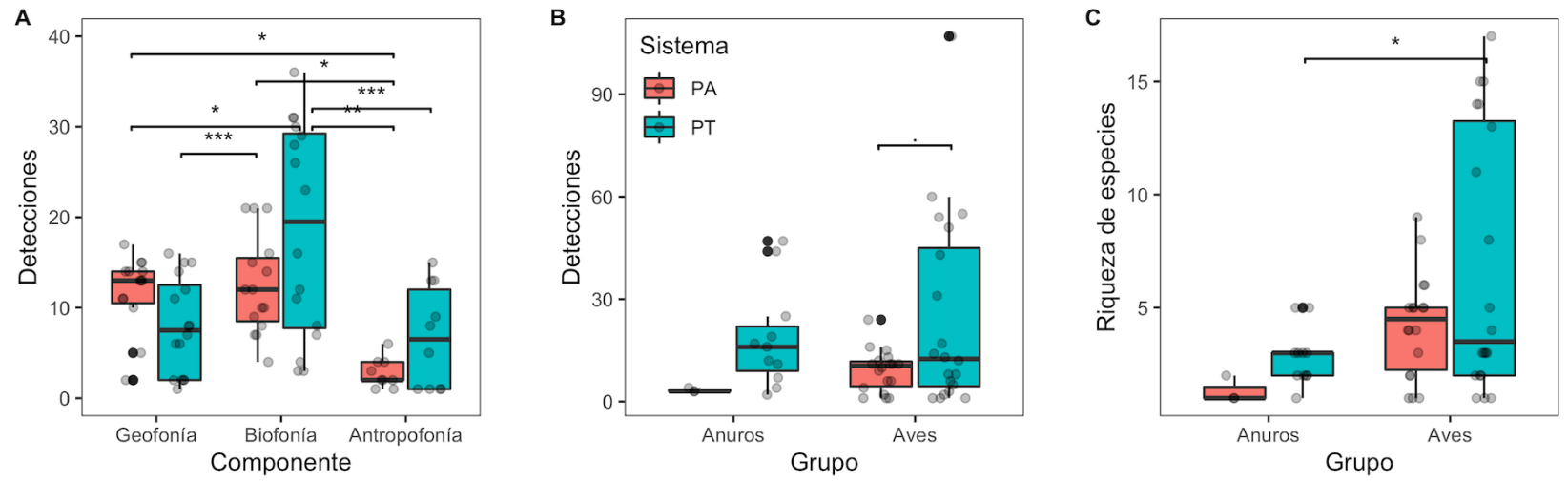

Figura 3. Variación del paisaje sonoro en potreros arbolados (PA) y potreros tradicionales (PT) en el departamento del Meta, Colombia. A, detecciones de componentes del paisaje sonoro (geofonía, biofonía o antropofonía); B, detecciones de anuros y aves; C, número de especies de anuros y aves reportadas por hora y sitio para sistemas silvopastoriles. Diagrama de cajas representa la mediana y el rango intercuartílico (IQR; $50 \%$ de los datos), el bigote (línea vertical) se extiende \pm 1.5 veces el IQR. Las líneas horizontales vinculan grupos significativamente diferentes (significancia: $[],. P>0.1 ;\left[{ }^{*}\right], P>0.05 ;\left[{ }^{* *}\right], P>$ $\left.\left.0.01 ;{ }^{* * *}\right], P>0.001\right)$

Figure 3. Variation of the soundscape in wooded pastures (PA) and traditional pastures (PT) in Meta department, Colombia. A, detections of the soundscape component (geophony, biophony or anthrophony); B, detections of anurans and birds; C, number of species of anurans and birds reported per hour and site. Box plot represents median and interquartile range (IQR; $50 \%$ of data), mustache (vertical line) extends \pm 1.5 times IQR. Horizontal lines link significantly different groups (significance: [.], $\left.P>0.1 ;\left[{ }^{*}\right], P>0.05 ;\left[{ }^{* *}\right], P>0.01 ;{ }^{* * *}\right], P>0.001$ )

Tabla 3. Efecto de sistema (PT, potreros tradicionales), componente del paisaje sonoro (B, biofonía; G, geofonía), grupo de aves (Av) e interacciones sobre las detecciones del componente sonoro, detecciones de anuros o aves y número de especies de anuros y aves, en el departamento del Meta, Colombia.

Table 3. System effect (PT, traditional pastures), soundscape component (B, biophony; G, geophony), group of birds (Av) and interactions on the detections of the sound component, detections of anurans or birds and number of species of anurans and birds in Meta department, Colombia.

\begin{tabular}{lcccccc}
\hline & \multicolumn{2}{c}{$\begin{array}{c}\text { Detecciones por com- } \\
\text { ponente sonoro }\end{array}$} & $\begin{array}{c}\text { Detecciones de anuros o } \\
\text { aves }\end{array}$ & \multicolumn{2}{c}{$\begin{array}{c}\text { Riqueza de especies de } \\
\text { anuros y aves }\end{array}$} \\
\hline & Coef \pm SE & $\mathrm{P}$ & Coef \pm SE & $\mathrm{P}$ & Coef \pm SE & $\mathrm{P}$ \\
\hline Intercepto & $1.02 \pm 0.20$ & $<0.001$ & $1.20 \pm 0.32$ & $<0.001$ & $0.29 \pm 0.50$ & 0.565 \\
\hline Sistema (PT) & $0.88 \pm 0.23$ & $<0.001$ & $1.72 \pm 0.32$ & $<0.001$ & $0.75 \pm 0.53$ & 0.159 \\
\hline Componente (B) & $1.50 \pm 0.21$ & $<0.001$ & - & - & - & - \\
\hline Grupo (Av) & - & - & $1.01 \pm 0.33$ & 0.002 & $1.15 \pm 0.51$ & 0.025 \\
\hline Componente (G) & $1.40 \pm 0.21$ & $<0.001$ & - & - & - & - \\
\hline Sistema (PT)*Componente (B) & $-0.48+0.25$ & 0.058 & - & - & - & - \\
\hline Sistema $(\mathrm{PT})^{*}$ Grupo (Av) & - & - & $-0.72 \pm 0.34$ & 0.032 & $-0.27 \pm 0.55$ & 0.631 \\
\hline Sistema $(\mathrm{PT})^{*}$ Componente $(\mathrm{G})$ & $-1.23 \pm 0.26$ & $<0.001$ & - & - & - & - \\
\hline
\end{tabular}


y aves (Figura 3C), la diferencia más marcada fue entre la riqueza de aves y anuros en potreros tradicionales (Figura 3c, $P=0.043$ ). Cinco de los seis modelos mejor ajustados incluyeron las variables distancia a vías y distancia a quebradas como las variables más importantes para explicar la variación de riqueza de estos dos grupos (Tabla S2, Figura S4). Aunque no se profundizó en la identificación de insectos acústicamente activos, se obtuvieron 237 detecciones (102 para potreros arbolados y 135 para potreros tradicionales).

Al revisar la lista de especies detectadas, se resalta la poca diversidad total reportada en potreros arbolados, con 2 especies de anuros y 23 especies de aves (Tabla 4, ver datos extendidos en Tabla S3). Por su parte, los potreros tradicionales registraron 5 especies de anuros y 30 especies de aves. La especie de anuro con más detecciones fue Dendropsophus mathiassoni, únicamente registrado en potreros tradicionales, principalmente al atardecer, seguido por Leptodactylus fuscus presente en ambos sistemas, pero con muchas más detecciones (59) en potrero tradicional. $\mathrm{El}$ ave con mayor número de detecciones fue Cyanocorax violaceus, seguido por Sturnella magna y Mesembrinibis cayennensis, registrados en ambos sistemas, pero mayoritariamente en potreros tradicionales. Este patrón se mantuvo para casi todas las especies, excepto Tyrannus melancholicus, Crypturellus cinereus, Pachyramphus polychopterus, Gymnomystax mexicanus, Ramphastos vitellinus y Machaeropterus striolatus (Tabla 4), que tuvieron más detecciones en potreros arbolados que en tradicionales.

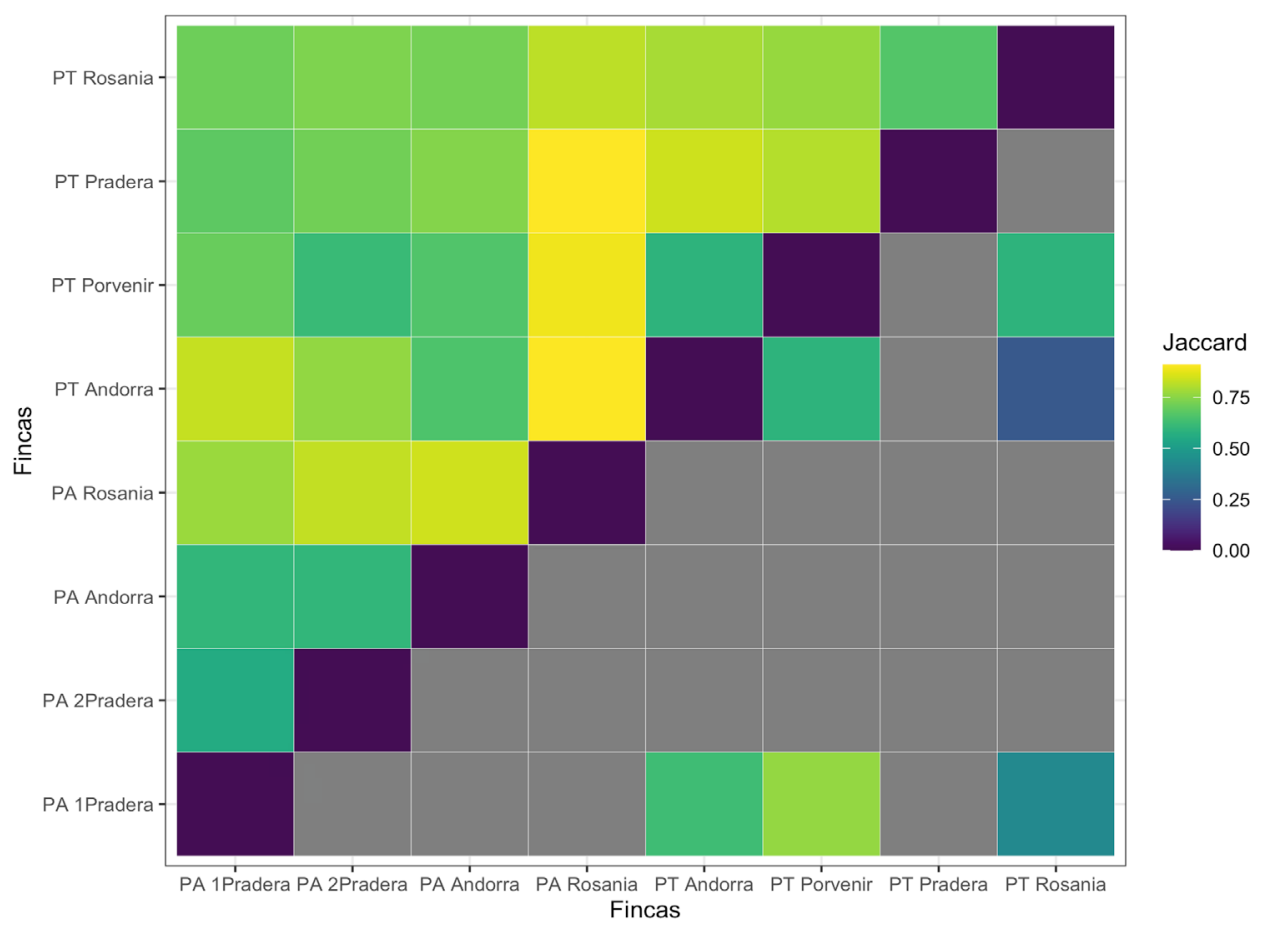

Figura 4. Matriz de disimilitud por pares entre las fincas muestreadas para sonidos de aves (arriba de la diagonal) y anuros (debajo de la diagonal) en el departamento del Meta, Colombia. El índice de Jaccard fue calculado a partir de la presencia de especies detectada en 587 grabaciones revisadas. Los valores más altos (más amarillos) indican una mayor disimilitud entre sitios pareados. Bloques grises representan sitios que no tuvieron presencia de anuros durante la revisión de coros de amanecer (5:00-8:00) o nocturno (17:00-20:00).

Figure 4. Pairwise dissimilarity matrix between the farms sampled for sounds of birds (above the diagonal) and anurans (below the diagonal) in Meta department, Colombia. The Jaccard index was calculated from the presence of species detected in 587 reviewed recordings. Higher values (more yellow) indicate greater dissimilarity between paired sites. Gray blocks represent sites that had no presence of anurans during the review of dawn (5:00-8:00) and dusk (17:00-20:00) choruses. 
Tabla 4. Listado de especies de anuros y aves registrados en coros de amanecer (AM: 05:00-08:00) y coro nocturno (PM: 17:0020:00) en potreros arbolados y tradicionales en el piedemonte llanero del departamento del Meta, Colombia. Los números indican el promedio de grabaciones con detecciones positivas por franja (AM y PM), así como el total de detecciones para cada sistema y general por especie.

Table 4. List of species of anurans and birds recorded in sunrise (AM: 05: 00-08: 00) and noctunal (PM: 17: 00-20: 00) choruses in wooded and traditional pastures in the foothills of the Meta department, Colombia. Numbers indicate the average of recordings with positive detections per time-group (AM and PM), as well as the total detections for each system and overall by species.

\begin{tabular}{|c|c|c|c|c|c|c|c|}
\hline \multirow{2}{*}{ Especies } & \multicolumn{3}{|c|}{ Potreros Arbolados } & \multicolumn{3}{|c|}{ Potreros Tradicionales } & \multirow{2}{*}{ Total } \\
\hline & AM & PM & Total & AM & PM & Total & \\
\hline \multicolumn{8}{|l|}{ Anuros } \\
\hline Dendropsophus mathiassoni & 0.0 & 0.0 & & 1.0 & 24.0 & 75 & 75 \\
\hline Leptodactylus fuscus & 0.0 & 1.3 & 4 & 2.7 & 18.3 & 63 & 67 \\
\hline Elachistocleis ovalis & 0.0 & 2.0 & 6 & 0.0 & 12.7 & 38 & 44 \\
\hline Leptodactylus colombiensis & 0.0 & 0.0 & & 5.3 & 2.7 & 24 & 24 \\
\hline Rhinella beebei & 0.0 & 0.0 & & 0.0 & 1.3 & 4 & 4 \\
\hline \multicolumn{8}{|l|}{ Aves } \\
\hline Cyanocorax violaceus & 16.7 & 4.3 & 63 & 24.0 & 10.0 & 102 & 165 \\
\hline Sturnella magna & 1.3 & 0.0 & 4 & 19.0 & 1.7 & 62 & 66 \\
\hline Mesembrinibis cayennensis & 5.0 & 1.3 & 19 & 10.0 & 3.0 & 39 & 58 \\
\hline Vanellus chilensis & 3.0 & 0.3 & 10 & 7.0 & 4.7 & 35 & 45 \\
\hline Cercomacroides tyrannina & 0.0 & 0.0 & & 12.7 & 1.3 & 42 & 42 \\
\hline Milvago chimachima & 0.0 & 0.0 & & 5.7 & 2.3 & 24 & 24 \\
\hline Tyrannus melancholicus & 4.0 & 1.3 & 16 & 2.3 & 0.0 & 7 & 23 \\
\hline Ara severus & 0.0 & 0.0 & & 4.3 & 3.0 & 22 & 22 \\
\hline Rupornis magnirostris & 1.7 & 0.0 & 5 & 4.0 & 1.7 & 17 & 22 \\
\hline Orthopsittaca manilatus & 0.3 & 0.3 & 3 & 3.7 & 2.3 & 18 & 21 \\
\hline Ortalis guttata & 2.0 & 0.0 & 6 & 4.3 & 0.3 & 14 & 20 \\
\hline Turdus leucomelas & 0.3 & 0.0 & 1 & 5.7 & 0.7 & 19 & 20 \\
\hline Amazona ochrocephala & 0.7 & 0.7 & 4 & 2.3 & 2.7 & 15 & 19 \\
\hline Syrigma sibilatrix & 2.7 & 0.3 & 9 & 3.0 & 0.3 & 10 & 19 \\
\hline Crypturellus cinereus & 2.3 & 1.3 & 11 & 1.0 & 0.7 & 5 & 16 \\
\hline Leistes militaris & 0.0 & 0.0 & & 4.3 & 0.7 & 15 & 15 \\
\hline Myrmophylax atrothorax & 1.0 & 0.0 & 3 & 1.7 & 0.0 & 5 & 8 \\
\hline Nyctidromus albicollis & 0.3 & 0.0 & 1 & 1.7 & 0.3 & 6 & 7 \\
\hline Eupsittula pertinax & 0.3 & 0.0 & 1 & 1.0 & 0.7 & 5 & 6 \\
\hline
\end{tabular}




\begin{tabular}{lccccccc}
\hline \multirow{2}{*}{ Especies } & \multicolumn{4}{c}{ Potreros Arbolados } & \multicolumn{2}{c}{ Potreros Tradicionales } & \multirow{2}{*}{ Total } \\
\cline { 2 - 7 } & AM & PM & Total & AM & PM & Total & \\
\hline Ictinia plumbea & 0.0 & 0.0 & & 1.0 & 1.0 & 6 & 6 \\
\hline Pitangus sulphuratus & 0.0 & 0.0 & & 2.0 & 0.0 & 6 & 6 \\
\hline Pteroglossus castanotis & 0.3 & 0.3 & 2 & 0.3 & 1.0 & 4 & 6 \\
\hline Legatus leucophaius & 0.0 & 0.0 & & 0.7 & 0.7 & 4 & 4 \\
\hline Camptostoma obsoletum & 0.0 & 0.0 & & 1.0 & 0.0 & 3 & 3 \\
\hline Crotophaga ani & 0.3 & 0.0 & 1 & 0.7 & 0.0 & 2 & 3 \\
\hline Pachyramphus polychopterus & 0.7 & 0.0 & 2 & 0.3 & 0.0 & 1 & 3 \\
\hline Cacicus cela & 0.3 & 0.0 & 1 & 0.3 & 0.0 & 1 & 2 \\
\hline Megascops choliba & 0.0 & 0.3 & 1 & 0.0 & 0.3 & 1 & 2 \\
\hline Gymnomystax mexicanus & 0.7 & 0.0 & 2 & 0.0 & 0.0 & & 2 \\
\hline Ramphastos vitellinus & 0.3 & 0.0 & 1 & 0.0 & 0.0 & & 1 \\
\hline Machaeropterus striolatus & 0.3 & 0.0 & 1 & 0.0 & 0.0 & & 1 \\
\hline Patagioenas cayennensis & 0.0 & 0.0 & & 0.3 & 0.0 & 1 & 1 \\
\hline Turdus ignobilis & 0.0 & 0.0 & & 0.3 & 0.0 & 1 & 1 \\
\hline Total & & & $\mathbf{1 7 4}$ & & & $\mathbf{6 9 6}$ & $\mathbf{8 7 0}$ \\
\hline
\end{tabular}

El índice de disimilitud de Jaccard fue más alto en las comparaciones del sitio PA_Rosania con los potreros tradicionales de PT_Pradera, PT_Andorra y PT_Porvenir (Figura 4). Esto indica que hay un mayor recambio entre estos sitios. Sin embargo, PA_Rosania presentó un recambio de especies considerable con relación a los potreros arbolados, como PA_2Pradera y PA_Andorra. Esta disimilitud ocurrió también en fincas de potreros tradicionales, como la comparación de las fincas Pradera y Andorra. La comunidad de anuros tuvo muy pocas detecciones para poder hacer comparaciones adecuadas en el recambio de especies, pero los valores de mayor disimilitud estuvieron entre PT_Porvenir y PA_1Pradera. Al contrario, la comparación pareada con menor recambio estuvo entre PT_Andorra y PT_Rosania.

\section{Discusión}

Los resultados en este estudio resaltan las diferencias en el paisaje sonoro y actividad acústica entre los sistemas de potreros arbolados y tradicionales, en particular relacionados con la distancia a las vías y a las quebradas. En el caso de los sistemas de potreros arbolados no hay una relación clara, pero para los potreros tradicionales, a menor distancia de quebradas, mayor el uso del espacio acústico y viceversa (Tabla S1). Las quebradas en el área de estudio están asociadas a parches de bosque y probablemente esto permita establecimiento de mayor número de especies acústicamente activas cerca de los potreros tradicionales. Uno de los potreros arbolados (PA_2Pradera) resultó muy similar a algunos potreros tradicionales (PT_Andorra, PT_Pradera), con mayor actividad acústica, lo cual refleja un patrón de zona abierta, donde el paisaje acústico es saturado por la actividad de insectos y anfibios, similar a lo encontrado en un estudio de sistemas productivos (pastos para ganado, cultivos de arroz) al norte de Colombia (Furumo \& Aide, 2019). Si bien los bosques tropicales intactos poseen mayor diversidad acústica que sistemas agroforestales o agrícolas (Burivalova et al., 2019), es importante tener en cuenta la importancia de la heterogeneidad espacial a la hora de conservar y mantener la diversidad en los sistemas agrícolas en paisajes dominados por humanos (Fahrig et al., 2011).

En cuanto a los componentes del paisaje sonoro y riqueza de grupos indicadores, también se encontraron diferencias entre los sistemas ganaderos tradicionales y sistemas silvopastoriles, en especial en el componente 
de biofonía, explicada por las aves. Aun así, otros componentes no analizados en detalle en este estudio pueden estar influenciando los paisajes sonoros del piedemonte de la Orinoquia (p. ej., insectos; Aide et al., 2017).

Contrario a lo que se esperaba, los sistemas de potreros arbolados presentaron menor riqueza de aves y anuros. Esto puede estar relacionado con la ya mencionada cercanía a quebradas con los relictos de bosque en potreros tradicionales, pero también con el diseño del sistema silvopatoril, que involucra árboles de falso yopo $(M$. trianae) de la misma edad, en ocasiones muy cercanos entre sí, lo cual podría considerarse un monocultivo de yopo. Sería ideal poder incrementar la heterogeneidad en los árboles sembrados y aumentar la distancia entre los mismos, como estrategia de mantenimiento de biodiversidad (Hendershot et al., 2020). Igualmente, integrar diseños que involucren diferentes estados sucesionales y por ende estructuras que permitan la colonización de otro tipo de biota en los sistemas silvopastoriles (Acevedo-Charry \& Aide, 2019). Dentro de las nuevas propuestas de sistemas agroforestales (silvopastoriles) para la conservación de la biodiversidad y soberanía alimentaria se plantea un manejo con palmas de regeneración ( $\mathrm{p}$. ej., Acronomia aculeata) y diferentes especies de árboles maderables (p. ej. Swietenia macrophylla, Erythrina, Inga, Ficus), lo cual podría ser una herramienta prometedora para aumentar la diversidad de especies leñosas nativas en tierras degradadas (Calle et al., 2017). Sin embargo, esto requiere esfuerzos de largo plazo y depende de los recursos e incentivos de programas estatales y globales, además de la disposición de los propietarios (Acevedo-Charry \& Aide, 2019).

Nuestros resultados mostraron una mayor detección de individuos de anuros en sistemas de potrero tradicional, lo cual posiblemente está determinado por la disponibilidad de cuerpos de agua temporales o permanentes, que son dispuestos en los sistemas tradicionales para permitir el acceso del ganado al agua, como bebederos artificiales. Igualmente, el pisoteo reiterado del ganado cambia las condiciones del suelo, generando pequeñas charchas que pueden ser aprovechadas por especies del género Leptodactylus (Angarita-Sierra, 2014). Esta opción puede ocurrir en la mayoría de las fincas que manejan más de 300 cabezas de ganado, a excepción de El Porvenir, que maneja 70-80 cabezas. Por ejemplo, $L$. fuscus y L. colombiensis depositan sus huevos en nidos de espuma que flotan en cuerpos de agua de sistemas lénticos y las larvas salen del nido a la charca (Crump, 1974). Las especies más abundantes en nuestro estudio,
D. mathiassoni, E. ovalis y R. beebei, tienen una estrategia reproductiva caracterizada por depositar huevos en cuerpos de agua lénticos, y sus larvas tienen desarrollo acuático (Crump, 1974). Adicionalmente, se ha documentado que algunas especies generalistas de anuros (Rhinella marina y Boana punctata) aprovechan los cuerpos de agua en áreas abiertas como medio de reproducción (Acevedo-Charry \& Aide, 2019).

Futuras aproximaciones al paisaje sonoro neotropical deben incluir detecciones de insectos (Aide et al., 2017; Bobryk et al., 2015; Furumo \& Aide, 2019), que ocupan un amplio rango de frecuencia en espectro audible (Aide et al., 2017). A pesar de que no fue evaluado cuantitativamente, en este estudio también se detectaron especies de mamíferos como Dactylomys dactylinus, especialistas de bambú (Emmons, 1981; LaVal, 1976; Reyes Díaz, 2019). Los registros de esta especie se encontraron asociados a tres potreros tradicionales (fincas Andorra, Rosania y Porvenir) y dos potreros arbolados (fincas Andorra, Rosania) cercanos a bosque y quebradas. Adicionalmente, se detectaron también mamíferos grandes y perseguidos por conflicto con ganaderos, como Panthera onca en potreros arbolados en la finca Rosania y Puma concolor en potrero tradicional en la finca Andorra, en ambos casos relativamente cerca a un bosque. Aunque ambas especies son nativas, de amplia distribución y generalistas en sus presas, la disponibilidad de presas y reducción de hábitat podría atraerlas a los sistemas productivos de ganadería (Garrote et al., 2017). Aun así, estos registros sugieren que los parches de bosque conservados en las fincas contribuyen a mantener posibles movimientos de estas especies dentro de los predios (Polisar et al., 2003) y postula posibles oportunidades de conservación en estos sistemas productivos en una escala regional.

La industria agrícola y ganadera seguirá recibiendo cada vez más presión para ser simultáneamente productiva y sostenible, así que la atención a tecnicas novedosas que apoyen sus decisiones de manejo seguirá siendo relevante. El uso de técnicas de monitoreo acústico muestra tener un gran potencial ya que puede ser fácilmente estandarizada y aplicada a gran escala para proveer indicadores del impacto y la sostenibilidad de la industria agrícola (Doohan et al., 2019). Es importante que se propicie que los sistemas silvopastoriles generen una estructura más heterogénea y se conviertan en hábitat para otras plantas y animales, de forma que ayuden a incrementar la biodiversidad y mantener diferentes procesos ecológicos vitales en los paisajes ganaderos (Jose et al., 2019; Murgueitio et al., 2013; Rivera et al., 2013). 


\section{Agradecimientos}

Agradecemos al Proyecto Ganadería Colombiana Sostenible y sus socios: Global Environment Facility (GEF), gobierno del Reino Unido, Grupo Banco Mundial, Federación Colombiana de Ganaderos (FEDEGAN), Centro para la Investigación en Sistemas de Producción Agropecuaria (CIPAV), Fondo Acción y The Nature Conservancy, por la coordinación de la ejecución de los recursos para el desarrollo del proyecto-consultoría STEP CO-TNC108529-CS-CLS. A la Universidad de los Llanos y todas sus dependencias, y especialmente a la Oficina de Proyección Social y la decanatura de la Facultad de Ciencias Básicas e Ingeniería. A los dueños o administradores de las fincas: El Porvenir, Clemente Eleazar Burgos; La Rosania, Humberto Castro; Hacienda La Andorra y La Pradera, José Felipe Sánchez. A todo el equipo de la consultoría por su apoyo en campo. The Nature Conservancy y Sieve Analytics Inc. gestionaron el almacenamiento y acceso para análisis de los datos en la plataforma ARBIMON II. A T. Mitchell Aide por el préstamo de las grabadoras.

\section{Referencias}

Acevedo-Charry, O., \& Aide, T. M. (2019). Recovery of amphibian, reptile, bird and mammal diversity during secondary forest succession in the tropics. Oikos, April, 1065-1078.

https:/ / doi.org/10.1111/oik.06252

Aide, T. M., Hernández-Serna, A., Campos-Cerqueira, M., Acevedo-Charry, O., \& Deichmann, J. L. (2017). Species richness (of insects) drives the use of acoustic space in the tropics. Remote Sensing, 9(11), 1-12.

https:/ / doi.org/10.3390/rs9111096

Angarita-Sierra, T. (2014). Diagnosis del estado de conservación del ensamble de anfibios y reptiles presentes en los ecosistemas de sabanas inundables de la cuenca del río Pauto, Casanare, Colombia. Revista de la Academia Colombiana de Ciencias Exactas, Físicas y Naturales, 38(146), 53-78.

Bobryk, C. W., Rega-Brodsky, C. C., Bardhan, S., Farina, A., He, H. S., \& Jose, S. (2015). A rapid soundscape analysis to quantify conservation benefits of temperate agroforestry systems using low-cost technology. Agroforestry Systems, 90(6), 997-1008.

https:/ / doi.org/10.1007/s10457-015-9879-6

Bormpoudakis, D., Sueur, J., \& Pantis, J. D. (2013). Spatial heterogeneity of ambient sound at the habitat type level: Ecological implications and applications. Landscape Ecology, 28(3), 495-506.

https:/ / doi.org/10.1007/s10980-013-9849-1
Burivalova, Z., Wahyudi, B., Boucher, T. M., Ellis, P., Truskinger, A., Towsey, M., Roe, P., Marthinus, D., Griscom, B., \& Game, E. T. (2019). Using soundscapes to investigate homogenization of tropical forest diversity in selectively logged forests. Journal of Applied Ecology, 56(11), 2493-2504.

Burnham, K. P., \& Anderson, D. R. (2004). Multimodel inference: Understanding AIC and BIC in model selection. Sociological Methods and Research, 33(2), 261-304.

https:/ / doi.org/10.1177/0049124104268644

Calle, Z., Giraldo, A. M., Cardozo, A., A., G., \& Murgueitio, E. (2017). Chapter 17: Enhancing biodiversity in Neotropical silvopastoral systems: Use of indigenous trees and palms. In Montagnini, F. (Ed.). Integrating Landscapes: Agroforestry for Biodiversity. Pp. 417-438. Springer International Publishing.

https:/ / doi.org/10.1007/978-3-319-69371-2_17

Calle, Z., Murgueitio, E., \& Chará, J. (2012). Integrating forestry, sustainable cattle-ranching and landscape restoration. Unasylva, 63(1), 31-40.

Campos-Cerqueira, M., \& Aide, T. M. (2016). Improving distribution data of threatened species by combining acoustic monitoring and occupancy modelling. Methods in Ecology and Evolution, 7(11), 1340-1348.

https:/ / doi.org/10.1111/2041-210X.12599

Campos-Cerqueira, M., \& Aide, T. M. (2017). Changes in the acoustic structure and composition along a tropical elevational gradient. Journal of Ecoacoustics, 1(December), PNCO7I.

https:// doi.org/10.22261/jea.pnco7i

Campos-Cerqueira, M., Mena, J. L., Tejeda-Gómez, V., Aguilar-Amuchastegui, N., Gutierrez, N., \& Aide, T. M. (2019). How does FSC forest certification affect the acoustically active fauna in Madre de Dios, Peru? Remote Sensing in Ecology and Conservation, 1-12.

https:/ / doi.org/10.1002/rse2.120

Crump, M. L. (1974). Reproductive strategies in a tropical anuran cominunity. Miscellaneous Publication, 61, 1-68.

Depraetere, M., Pavoine, S., Jiguet, F., Gasc, A., Duvail, S., \& Sueur, J. (2012). Monitoring animal diversity using acoustic indices: Implementation in a temperate woodland. Ecological Indicators, 13(1), 46-54.

https:/ / doi.org/10.1016/j.ecolind.2011.05.006

Doohan, B., Fuller, S., Parsons, S., \& Peterson, E. E. (2019). The sound of management: Acoustic monitoring for agricultural industries. Ecological Indicators, 96(September 2018), 739-746.

https:/ / doi.org/10.1016/j.ecolind.2018.09.029 
Dymond, C. C., Mladenoff, D. J., \& Radeloff, V. C. (2002). Phenological differences in Tasseled Cap indices improve deciduous forest classification. Remote Sensing of Environment, 80(3), 460-472.

https:/ / doi.org/10.1016/S0034-4257(01)00324-8

Emmons, L. H. (1981). Morphological, ecological, and behavioral adaptations for arboreal browsing in Dactylomys dactylinus (Rodentia, Echimyidae). Journal of Mammalogy, 62(1), 183-189.

Fahrig, L., Baudry, J., Brotons, L., Burel, F. G., Crist, T. O., Fuller, R. J., Sirami, C., Siriwardena, G. M., \& Martin, J. (2011). Functional landscape heterogeneity and animal biodiversity in agricultural landscapes. Ecology Letters, 14(2), 101-112.

Fajardo, D., Johnston González, R., Neira, L., Chará, J., \& Murgueitio, E. (2009). Influencia de sistemas silvopastoriles en la diversidad de aves en la cuenca del río La Vieja, Colombia. Recursos Naturales y Ambiente (CATIE), 58, 9-16.

Furumo, P. R., \& Aide, T. M. (2019). Using soundscapes to assess biodiversity in Neotropical oil palm landscapes. Landscape Ecology, 34(4), 911-923.

https:/ / doi.org/10.1007/ s10980-019-00815-w

Garrote, G., Rodríguez-Castellanos, F., Trujillo, F., \& Mosquera-Guerra, F. (2017). Características de los ataques de jaguar (Panthera onca) sobre el ganado y evaluación económica de las pérdidas en fincas ganaderas de los Llanos Orientales (Vichada, Colombia). En Casdtaño-Uribe, C. et al. (Eds.). II. Conflicto Entre Felinos y Humanos en América Latina. Pp. 89-102. Instituto Alexander von Humboldt, Fundación Herencia Ambiental Caribe, Fundación Panthera, Bogotá.

Gibb, R., Browning, E., Glover-Kapfer, P., \& Jones, K. E. (2019). Emerging opportunities and challenges for passive acoustics in ecological assessment and monitoring. Methods in Ecology and Evolution, 10(2), 169-185.

https:/ / doi.org/10.1111/2041-210X.13101

Hendershot, J. N., Smith, J. R., Anderson, C. B., Letten, A. D., Frishkoff, L. O., Zook, J. R., Fukami, T., \& Daily, G. C. (2020). Intensive farming drives longterm shifts in avian community composition. Nature, 579(7799), 393-96.

https:/ / doi.org/10.1038/s41586-020-2090-6

Herrera-Montes, M. I. (2018). Protected area zoning as a strategy to preserve natural soundscapes, reduce anthropogenic noise intrusion, and conserve biodiversity. Tropical Conservation Science, 11(1).

https:/ / doi.org/10.1177/1940082918804344

IDEAM. (2005). Atlas climatológico de Colombia [en línea].
IDEAM. (2019). Décimo séptimo Boletín de Detecciones Tempranas de Deforestación.

http:// www.ideam.gov.co/documents / 24277/ 84382637/Detecciones+Tempranas+de+Deforestación/96e81976-195e-4d0f-8aaf-24c05c7312f8

Jaccard, P. (1912). The distribution of the flora in the Alpine zone. New Phytologist, 11(2), 37-50.

https:/ / doi.org/10.1111/j.1469-8137.1912.tb05611.x

Joo, W., Gage, S. H., \& Kasten, E. P. (2011). Analysis and interpretation of variability in soundscapes along an urban-rural gradient. Landscape and Urban Planning, 103(3-4), 259-276.

https:/ / doi.org/10.1016/j.landurbplan.2011.08.001

Jose, S., Walter, D., \& Mohan Kumar, B. (2019). Ecological considerations in sustainable silvopasture design and management. Agroforestry Systems, 93(1), 317-331.

https:/ / doi.org/10.1007/s10457-016-0065-2

Kalan, A. K., Mundry, R., Wagner, O. J. J., Heinicke, S., Boesch, C., \& Kühl, H. S. (2015). Towards the automated detection and occupancy estimation of primates using passive acoustic monitoring. Ecological Indicators, 54, 217-226.

https:/ / doi.org/10.1016/j.ecolind.2015.02.023

LaVal, R. K. (1976). Voice and habitat of Dactylomys dactylinus (Rodentia: Echimyidae) in Ecuador. Journal of Mammalogy, 57(2), 402-404.

Manakin, R. W., Hagstrum, D. W., Smith, M. ., Roda, A. L., \& Kairo, M. T. K. (2011). Perspective and promise : A century of insect acoustic detection and monitoring. American Entomologist, 57(1), 30-44.

https:/ / doi.org/10.1093/ae/57.1.30

Murgueitio, E., Chará, J., Solarte, A. J., Uribe, F., Zapata, C., \& Rivera, J. E. (2013). Agroforestería pecuaria y sistemas silvopastoriles intensivos (SSPi) para la adaptación ganadera al cambio climático con sostenibilidad. Revista Colombiana de Ciencias Pecuarias, 26, 313-316.

Naranjo, L. G. (1992). Estructura de la avifauna en un área ganadera en el Valle del Cauca, Colombia. Caldasia, 17(1), 55-66.

Oksanen, J., Blanchet, F. G., Michael, F., Kindt, R., Legendre, P., McGlinn, D., Minchin, P. R., O'Hara, R. B., Simpson, G. L., \& Solymos, P. (2019). Vegan: Community Ecology Package. R package version 2.5-5.

Ospina, O. E., Villanueva-Rivera, L. J., Corrada-Bravo, C. J., \& Mitchell Aide, T. (2013). Variable response of anuran calling activity to daily precipitation and temperature: Implications for climate change. Ecosphere, 4(4), 1-12.

https:/ / doi.org/10.1890/ES12-00258.1 
Pekin, B. K., Jung, J., Villanueva-Rivera, L. J., Pijanowski, B. C., \& Ahumada, J. A. (2012). Modeling acoustic diversity using soundscape recordings and LIDAR-derived metrics of vertical forest structure in a neotropical rainforest. Landscape Ecology, 27(10), 1513-1522. https:/ / doi.org/10.1007/s10980-012-9806-4

Pijanowski, B. C., Villanueva-Rivera, L. J., Dumyahn, S. L., Farina, A., Krause, B. L., Napoletano, B. M., Gage, S. H., \& Pieretti, N. (2011). Soundscape ecology: The science of sound in the Landscape. BioScience, 61(3), 203-216.

https:/ / doi.org/10.1525/bio.2011.61.3.6

Polisar, J., Maxit, I., Scognamillo, D., Farrell, L., Sunquist, M. E., \& Eisenberg, J. F. (2003). Jaguars, pumas, their prey base, and cattle ranching: ecological interpretations of a management problem. Biological Conservation, 109(2), 297-310.

Reyes Díaz, M. C. (2019). Aproximación etológica de Dactylomys dactylimus (RODENTIA: Echymyidae-Desmarest, 1871) en la Universidad de los Llanos. Tesis. Villavicencio: Departamento de Biologío y Química, Universidad de los Llanos. 103 pp.

Rivera, L. F., Armbrecht, I., \& Calle, Z. (2013). Silvopastoral systems and ant diversity conservation in a cattle-dominated landscape of the Colombian Andes. Agriculture, Ecosystems and Environment, 181, 188-194.

https:/ / doi.org/10.1016/j.agee.2013.09.011

Rodríguez-Buriticá, S., Savageb, D., Caycedo, P., Acevedo-Charrya, O., Isazad, C., Daza, J. M., Almeirae, J., Ulloaf, J. S., Orozco-Alzateh, M., Villamizari, N., José Francisco Ruizh, J., Loperak, A., Oliverl, B., Laverdem, Ó., Pijanowski, B., \& Colombiana, R. E. (2018). Paisajes sonoros de Colombia: La otra dimensión de la biodiversidad. Ficha 103 para el Reporte de Estado y Tendencias de la Biodiversidad Continental de Colombia 2018. Ficha 103. Bogotá D. C: Instituto de Investigación de Recursos Biológicos Alexander von Humboldt Disponible en

https:/ / reporte.humboldt.org.co/biodiversi$\mathrm{dad} / 2018 / \mathrm{cap} 1 / 103 /$
Romero-Ruiz, M. H., Flantua, S. G. A., Tansey, K., \& Berrío, J. C. (2012). Landscape transformations in savannas of northern South America: Land use/cover changes since 1987 in the Llanos Orientales of Colombia. Applied Geography, 32(2), 766-776.

https:/ / doi.org/10.1016/j.apgeog.2011.08.010

Samarawickrama, U., Piyaratne, D., \& Ranagalage, M. (2017). Relationship between NDVI with Tasseled cap indices : A remote sensing based analysis. International Journal of Innovative Research in Technology, 3(12), 13-19.

Sueur, J., Aubin, T., \& Simonis, C. (2008). Sound analysis and synthesis with the package Seewave, a free modular tool for sound. Bioacoustics, 18(2), 213-226.

https:/ / doi.org/10.1080/09524622.2008.9753600

Tucker, D., Gage, S. H., Williamson, I., \& Fuller, S. (2014). Linking ecological condition and the soundscape in fragmented Australian forests. Landscape Ecology, 29(4), 745-758.

https:/ / doi.org/10.1007/s10980-014-0015-1

Vrieling, A., Meroni, M., Darvishzadeh, R., Skidmore, A. K., Wang, T., Zurita-Milla, R., Oosterbeek, K., O'Connor, B., \& Paganini, M. (2018). Vegetation phenology from Sentinel-2 and field cameras for a Dutch barrier island. Remote Sensing of Environment, 215(March 2018), 517-529.

https:/ / doi.org/10.1016/j.rse.2018.03.014

Williams, B. A., Grantham, H. S., Watson, J. E. M., Alvarez, S. J., Simmonds, J. S., Rogéliz, C. A., Da Silva, M., Forero-Medina, G., Etter, A., \& Nogales, J. (2020). Minimising the loss of biodiversity and ecosystem services in an intact landscape under risk of rapid agricultural development. Environmental Research Letters, 15(1), 14001.

$\mathrm{Wu}, \mathrm{J}$. (2004). Effects of changing scale on landscape pattern analysis: Scaling relations. Landscape Ecology, 19(2), 125-138.

https://doi.org/10.1023/b:land.0000021711.40074.ae 


\section{Información suplementaria}

A

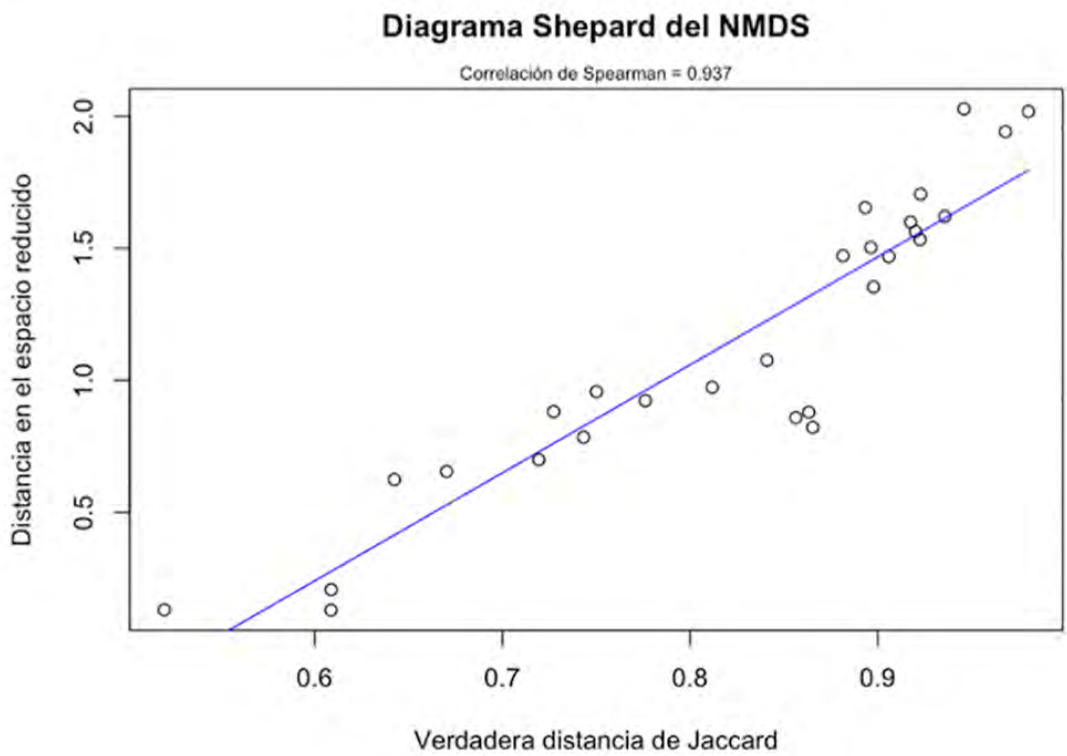

B

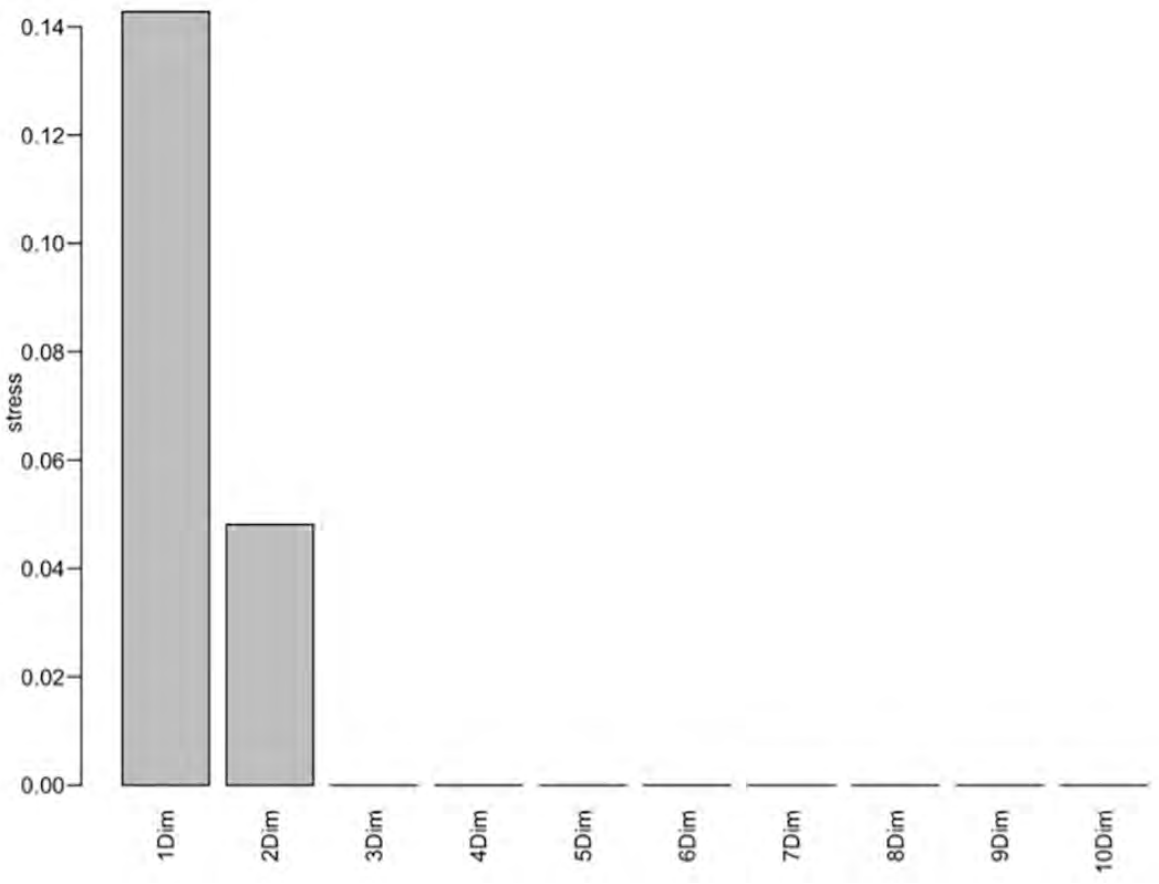

Figura S1. Estudio de paisajes sonoros en el departamento del Meta, Colombia. A, diagrama de Shepard para verificación y visualización de calidad en la representación en dos dimensiones para NMDS; B, gráfico de barras del valor de estrés de la ordenación para diferentes dimensiones (nótese que después de dos dimensiones el estrés declina drásticamente). 


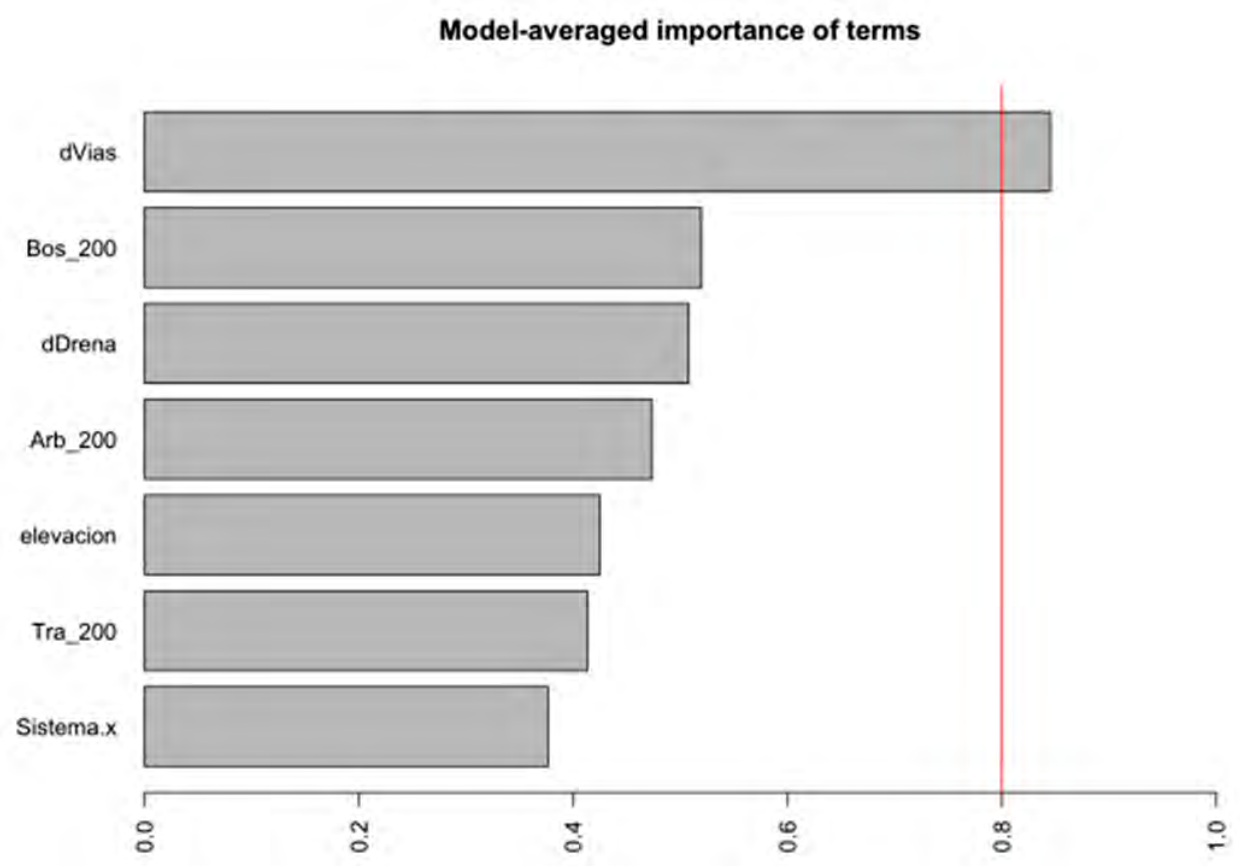

Figura S2. Estudio de paisajes sonoros en el departamento del Meta, Colombia. Importancia de variables en selección de modelos para detecciones de componente de paisaje sonoro.

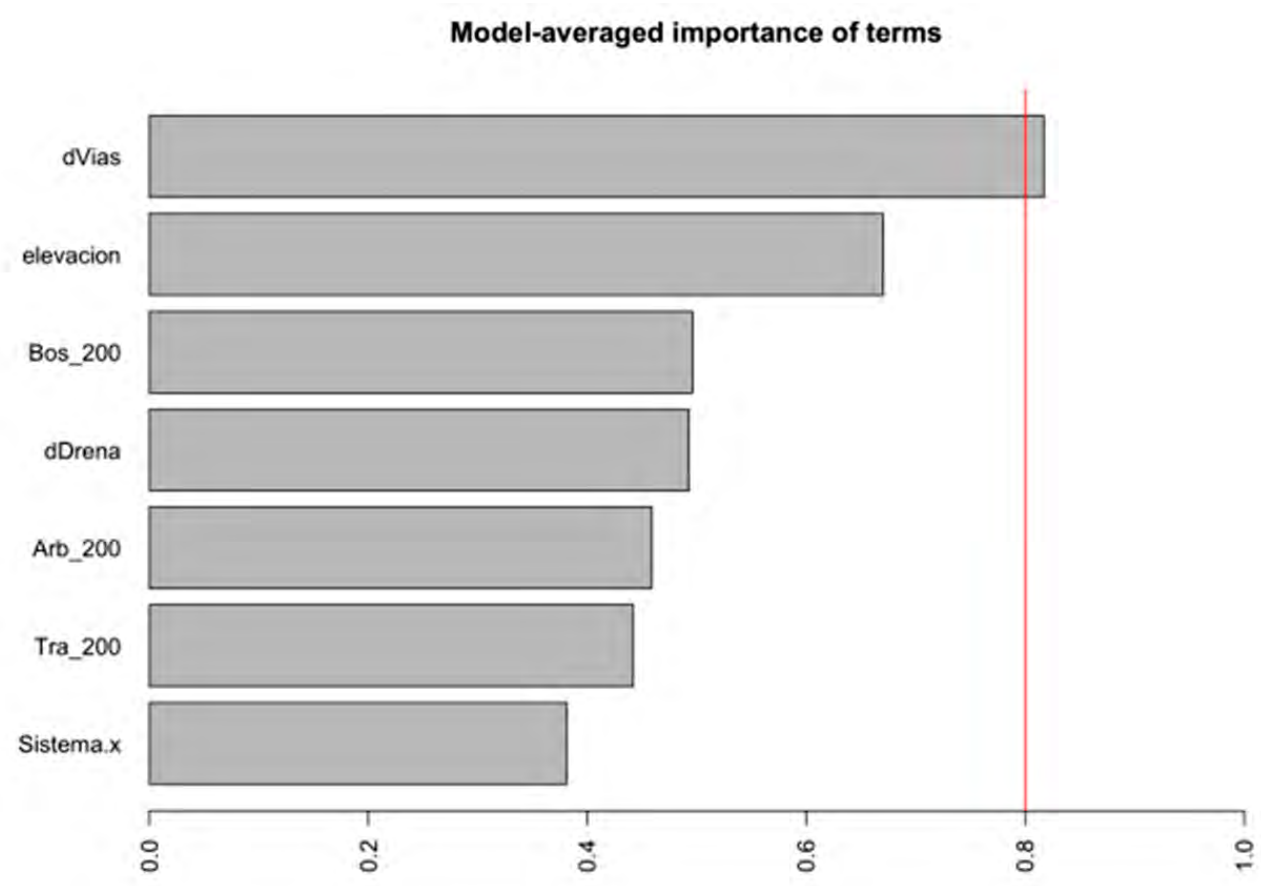

Figura S3. Estudio de paisajes sonoros en el departamento del Meta, Colombia. Importancia de variables en selección de modelos para detecciones de aves y anuros. 


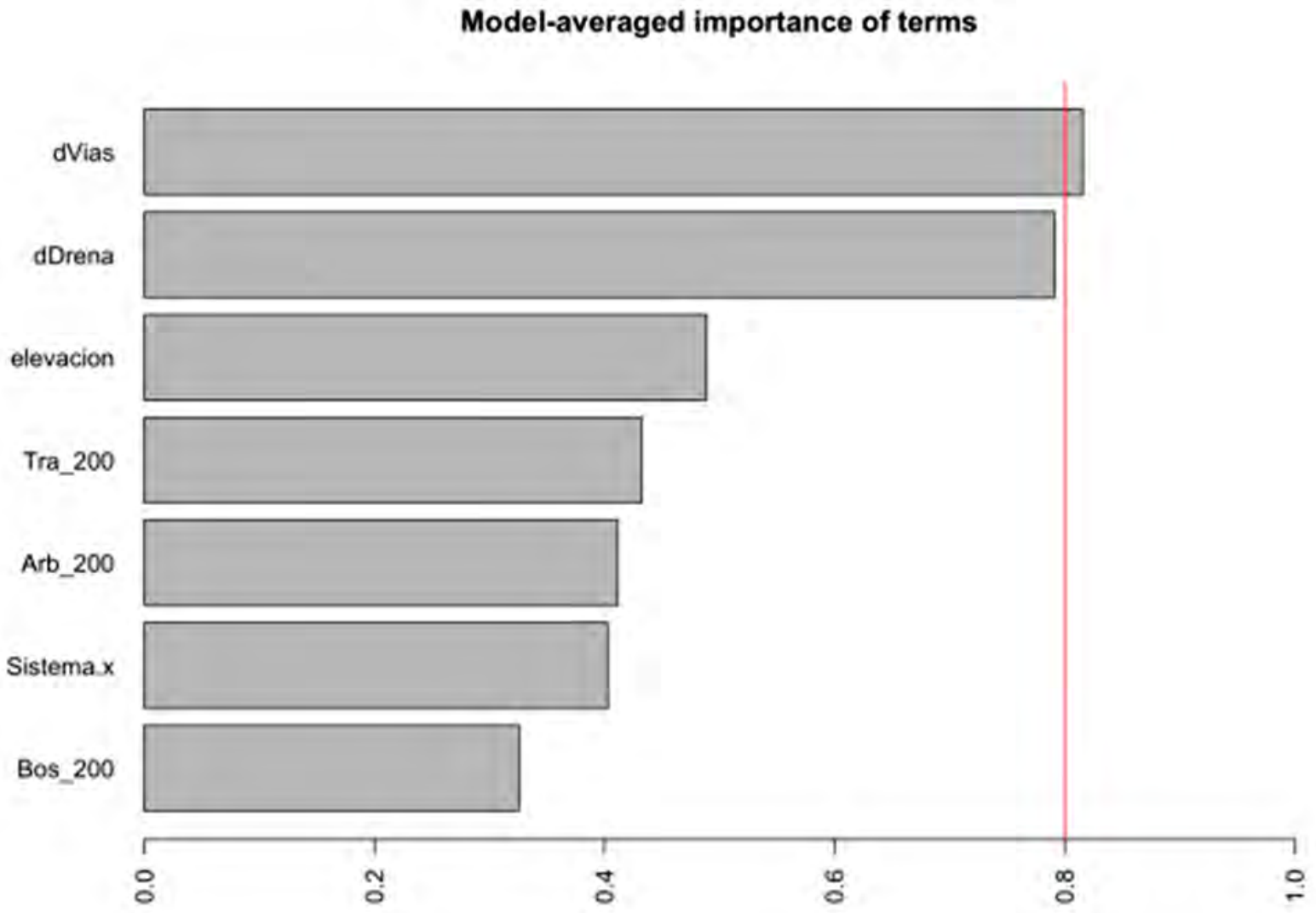

Figura S4. Estudio de paisajes sonoros en el departamento del Meta, Colombia. Importancia de variables en selección de modelos para riqueza de aves y anuros 


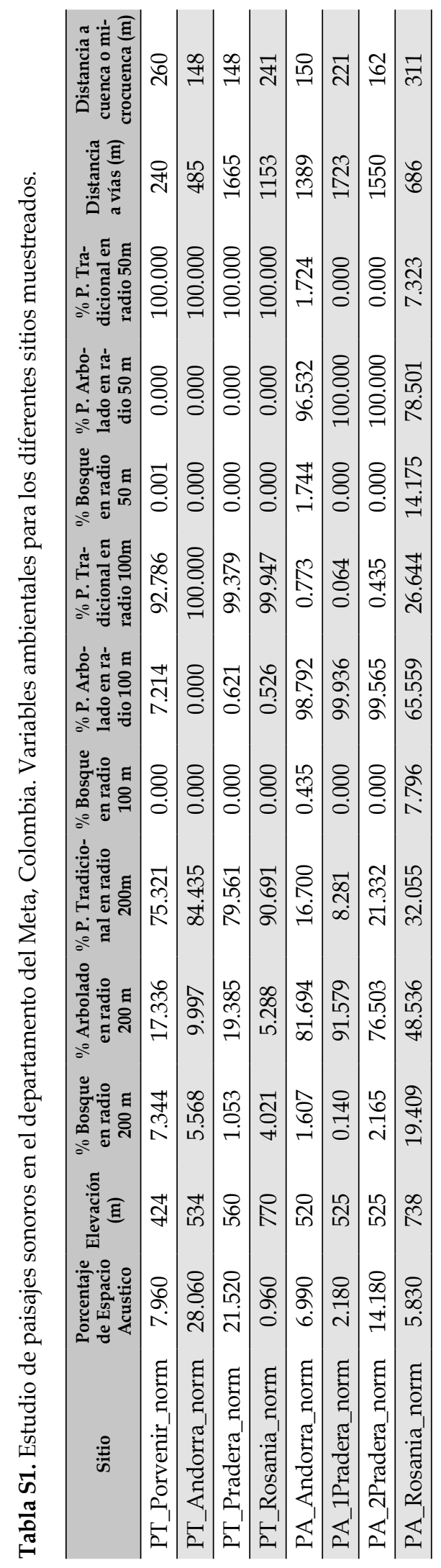


Tabla S2. Estudio de paisajes sonoros en el departamento del Meta, Colombia. Modelos mas posibles $(\triangle \mathrm{AICc}<2)$ para variables explicativas de tres variables respuesta.

\begin{tabular}{|c|c|c|c|}
\hline Variable respuesta & Modelo & AICc & Peso \\
\hline Detecciones Componente & freq $\sim 1+$ Bos_200 + dVias + dDrena & 542.479 & 0.053 \\
\hline Detecciones Componente & freq $\sim 1+$ Sistema. $x+$ elevacion + Arb_200 + dVias & 542.564 & 0.051 \\
\hline Detecciones Componente & freq $\sim 1+\mathrm{dVias}$ & 543.480 & 0.032 \\
\hline Detecciones Componente & freq $\sim 1+$ Sistema. $x+$ Bos_200 + dVias + dDrena & 543.592 & 0.031 \\
\hline Detecciones Componente & freq 1 + Bos_200 + Arb_200 + dVias + dDrena & 543.724 & 0.029 \\
\hline Detecciones Componente & freq $\sim 1+$ Bos_200 + Tra_200 + dVias + dDrena & 543.724 & 0.029 \\
\hline Detecciones Componente & freq $\sim 1+$ Bos $\_200+$ Arb $\_200+$ Tra $\_200+$ dVias + dDrena & 543.724 & 0.029 \\
\hline Detecciones Componente & freq $\sim 1+$ Arb_200 + Tra_200 + dVias + dDrena & 543.724 & 0.029 \\
\hline Detecciones Grupo & freq $\sim 1+$ elevacion + Bos $\_200+$ dVias & 379.849 & 0.050 \\
\hline Detecciones Grupo & freq $\sim 1+$ elevacion + Tra $\_200+$ dVias & 380.301 & 0.040 \\
\hline Detecciones Grupo & freq $\sim 1+$ Sistema. $x+$ elevacion + dVias & 380.503 & 0.036 \\
\hline Detecciones Grupo & freq $\sim 1+$ Sistema. $x+$ Arb_200 + dVias + dDrena & 380.544 & 0.036 \\
\hline Detecciones Grupo & freq $\sim 1+$ elevacion $+\mathrm{dVias}+\mathrm{dDrena}$ & 380.789 & 0.032 \\
\hline Detecciones Grupo & freq $\sim 1+$ elevacion + Bos_200 + dVias + dDrena & 380.810 & 0.031 \\
\hline Detecciones Grupo & freq $\sim 1+$ elevacion + Arb_200 + dVias & 380.866 & 0.030 \\
\hline Detecciones Grupo & freq $\sim 1+$ Sistema. $x+$ Tra $\_200+$ dVias + dDrena & 380.915 & 0.030 \\
\hline Detecciones Grupo & freq $\sim 1+$ Bos_200 + dVias + dDrena & 381.457 & 0.023 \\
\hline Detecciones Grupo & freq $\sim 1+$ elevacion + Bos_200 + Arb_200 + dVias & 381.581 & 0.021 \\
\hline Detecciones Grupo & freq $\sim 1+$ elevacion + Bos_200 + Arb_200 + Tra_200 + dVias & 381.581 & 0.021 \\
\hline Detecciones Grupo & freq $\sim 1+$ elevacion + Bos_200 + Tra_200 + dVias & 381.581 & 0.021 \\
\hline Detecciones Grupo & freq $\sim 1+$ elevacion + Arb_200 + Tra_200 + dVias & 381.581 & 0.021 \\
\hline Detecciones Grupo & freq $\sim 1+$ Bos_200 + dVias & 381.627 & 0.021 \\
\hline Detecciones Grupo & freq $~ 1+$ Arb_200 + Tra_200 + dVias + dDrena & 381.705 & 0.020 \\
\hline Detecciones Grupo & freq $~ 1+$ Bos $\_200+$ Tra $\_200+$ dVias + dDrena & 381.705 & 0.020 \\
\hline Detecciones Grupo & freq $\sim 1+$ Bos_200 + Arb_200 + dVias + dDrena & 381.705 & 0.020 \\
\hline Detecciones Grupo & freq $\sim 1+$ Bos_200 + Arb_200 + Tra_200 + dVias + dDrena & 381.705 & 0.020 \\
\hline Detecciones Grupo & freq $\sim 1+$ Sistema. $x+$ elevacion + Bos_200 + dVias & 381.735 & 0.020 \\
\hline Detecciones Grupo & freq $\sim 1+$ Sistema. $x+$ elevacion + dVias + dDrena & 381.755 & 0.019 \\
\hline Riqueza Grupo & freq $\sim 1+$ Arb_200 + dVias + dDrena & 248.937 & 0.086 \\
\hline Riqueza Grupo & freq $\sim 1+$ Tra_200 + dVias + dDrena & 249.180 & 0.076 \\
\hline Riqueza Grupo & freq $\sim 1+$ elevacion $+\mathrm{dVias}+\mathrm{dDrena}$ & 249.389 & 0.068 \\
\hline Riqueza Grupo & freq $\sim 1+$ Sistema. $x+d$ Vias + dDrena & 249.808 & 0.055 \\
\hline Riqueza Grupo & freq $\sim 1+\mathrm{dVias}+\mathrm{dDrena}$ & 249.973 & 0.051 \\
\hline Riqueza Grupo & freq $\sim 1+$ Sistema. $x+$ elevacion + Tra $\_200$ & 250.712 & 0.035 \\
\hline
\end{tabular}


Tabla S3. Estudio de paisajes sonoros en el departamento del Meta, Colombia. Lista de especies de anuros y aves con detecciones para cada especie (número de grabaciones).

\begin{tabular}{|c|c|c|c|c|c|c|c|c|c|c|c|c|c|c|c|c|c|c|c|}
\hline \multirow[b]{2}{*}{ Especies } & \multicolumn{9}{|c|}{ Potreros Arbolados } & \multicolumn{9}{|c|}{ Potreros Tradicionales } & \multirow[b]{2}{*}{ Total } \\
\hline & 5 & 6 & 7 & $\begin{array}{c}\text { AM } \\
\text { Prom }\end{array}$ & 17 & 18 & 19 & $\begin{array}{c}\text { PM } \\
\text { Prom }\end{array}$ & Total & 5 & 6 & 7 & $\begin{array}{c}\text { AM } \\
\text { Prom }\end{array}$ & 17 & 18 & 19 & $\begin{array}{c}\text { PM } \\
\text { Prom }\end{array}$ & Total & \\
\hline Anuros & & & & 0 & 0 & 0 & 0 & 2 & & 0 & 0 & 0 & 3 & & & & 5 & & \\
\hline Dendropsophus mathiassoni & 0 & 0 & 0 & 0,0 & 0 & 0 & 0 & 0,0 & & 3 & 0 & 0 & 1,0 & 4 & 32 & 36 & 24,0 & 75 & 75 \\
\hline Leptodactylus fuscus & 0 & 0 & 0 & 0,0 & 3 & 1 & ( & 1,3 & 4 & 8 & 0 & 0 & 2,7 & 18 & 22 & 15 & 18,3 & 63 & 67 \\
\hline Elachistocleis ovalis & 0 & 0 & 0 & 0,0 & 0 & 3 & 3 & 2,0 & 6 & 0 & 0 & 0 & 0,0 & 8 & 16 & 14 & 12,7 & 38 & 44 \\
\hline Leptodactylus colombi & 0 & 0 & 0 & 0,0 & 0 & 0 & 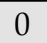 & 0,0 & & 14 & 0 & 2 & 5,3 & & 1 & 7 & 2,7 & 24 & 24 \\
\hline Rhinella beebei & 0 & 0 & 0 & 0,0 & 0 & 0 & & 0,0 & & 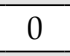 & 0 & 0 & 0,0 & & & & 1,3 & 4 & 4 \\
\hline Aves & & & & 20 & & & & 10 & & & & & 29 & & & & 21 & & \\
\hline Cyanocorax violaceus & 9 & 26 & 15 & 16,7 & 13 & 0 & 0 & 4,3 & 63 & 16 & 35 & 21 & 24,0 & 30 & 0 & 0 & 10,0 & 102 & 165 \\
\hline Sturnella magna & 0 & 2 & 2 & 1,3 & 0 & 0 & 0 & 0,0 & & 12 & 32 & 13 & 19,0 & 5 & & 0 & 1,7 & 62 & 66 \\
\hline Mesembrinibis & 5 & 5 & 5 & 5,0 & 3 & 1 & 0 & 1,3 & 1 & 9 & 10 & 11 & 10,0 & 9 & 0 & 0 & 3,0 & 39 & 58 \\
\hline Vanellus chilensis & 5 & 0 & 4 & 3,0 & 0 & 1 & 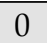 & 0,3 & 10 & 5 & 9 & 7 & 7,0 & 12 & 2 & 0 & 4,7 & 35 & 45 \\
\hline Cercomacroides tyra & 0 & 0 & 0 & 0,0 & ( & 0 & ( & 0,0 & & 8 & 23 & 7 & 12,7 & 4 & & & 1,3 & 42 & 42 \\
\hline Milv & 0 & 0 & 0 & 0,0 & 0 & 0 & 0 & 0,0 & & 4 & 12 & 1 & 5,7 & 7 & 0 & 0 & 2,3 & 24 & 24 \\
\hline Tyrannus me & 5 & 4 & 3 & 4,0 & 4 & 0 & 0 & 1,3 & 16 & 2 & 3 & 2 & 2,3 & 0 & 0 & 0 & 0,0 & 7 & 23 \\
\hline Ara severus & 0 & 0 & 0 & 0,0 & ( & 0 & & 0,0 & & 5 & 7 & 1 & & 9 & & & 3,0 & 22 & 22 \\
\hline Rupor & 0 & 2 & 3 & 1,7 & 0 & 0 & & 0,0 & 5 & 0 & 7 & 5 & 4,0 & 5 & 0 & 0 & 1,7 & 17 & 22 \\
\hline Orthopsittace & 1 & 0 & 0 & 0,3 & 1 & 0 & 0 & 0,3 & 3 & 5 & 5 & 1 & 3,7 & 7 & 0 & 0 & 2,3 & 18 & 21 \\
\hline Ortalis gu & 1 & 2 & 3 & 2,0 & ( & 0 & & 0,0 & & 3 & 8 & 2 & 4,3 & 1 & & 0 & 0,3 & 14 & 20 \\
\hline Turd & 1 & 0 & 0 & 0,3 & 0 & 0 & ( & 0,0 & 1 & 10 & 6 & 1 & 5,7 & 2 & 0 & 0 & 0,7 & 19 & 20 \\
\hline Amazo & 1 & 1 & 0 & 0,7 & 2 & 0 & 0 & 0,7 & 4 & 1 & 4 & 2 & 2,3 & 8 & 0 & 0 & 2,7 & 15 & 19 \\
\hline Syrig & 2 & 4 & 2 & 2,7 & 1 & 0 & & 0, & 9 & 3 & 4 & 2 & 3, & 1 & 0 & 0 & 0,3 & 10 & 19 \\
\hline Crypt & 0 & 3 & 4 & 2,3 & 3 & 1 & ( & 1,3 & 11 & 2 & 1 & 0 & 1,0 & 2 & 0 & 0 & 0,7 & 5 & 16 \\
\hline Leistes militaris & 0 & 0 & 0 & 0,0 & 0 & 0 & 0 & 0,0 & & 0 & 7 & 6 & 4, & 2 & 0 & 0 & 0,7 & 15 & 15 \\
\hline Myrm & 0 & 3 & 0 & 1,0 & 0 & 0 & 0 & 0,0 & 3 & 2 & 3 & 0 & 1,7 & 0 & 0 & 0 & 0,0 & 5 & 8 \\
\hline Nyctidro & 1 & 0 & 0 & 0,3 & 0 & 0 & 0 & 0,0 & 1 & 5 & 0 & 0 & 1,7 & 0 & 0 & 1 & 0,3 & 6 & 7 \\
\hline Eupsittul & 0 & 0 & 1 & 0,3 & 0 & 0 & 0 & 0,0 & 1 & 1 & 2 & 0 & $1,($ & 2 & 0 & 0 & 0,7 & 5 & 6 \\
\hline Ictinia & 0 & 0 & 0 & 0,0 & 0 & 0 & 0 & 0,0 & & 1 & 2 & 0 & 1,0 & 3 & 0 & 0 & 1,0 & 6 & 6 \\
\hline Pitangus & 0 & 0 & 0 & 0,0 & 0 & 0 & 0 & 0,0 & & 0 & 2 & 4 & 2,0 & 0 & 0 & 0 & 0,0 & 6 & 6 \\
\hline Pteroglos & 1 & 0 & 0 & 0,3 & 1 & 0 & 0 & 0,3 & 2 & 0 & 1 & 0 & & 3 & 0 & 0 & 1,0 & 4 & 6 \\
\hline Legatus leucophaius & 0 & 0 & 0 & 0,0 & 0 & 0 & 0 & 0,0 & & 0 & 1 & 1 & 0,7 & 1 & 1 & 0 & 0,7 & 4 & 4 \\
\hline Camptost & 0 & 0 & 0 & 0,0 & 0 & 0 & 0 & 0,0 & & 0 & 3 & 0 & 1,0 & 0 & 0 & 0 & 0,0 & 3 & 3 \\
\hline Crotopha & 0 & 0 & 1 & 0,3 & 0 & 0 & 0 & 0,0 & 1 & 0 & 2 & 0 & 0,7 & 0 & 0 & 0 & 0,0 & 2 & 3 \\
\hline Pachyramphus polycho & 0 & 2 & 0 & 0,7 & 0 & 0 & 0 & 0,0 & 2 & 0 & 0 & 1 & 0,3 & 0 & 0 & 0 & 0,0 & 1 & 3 \\
\hline Cacicus cela & 0 & 0 & 1 & 0,3 & 0 & 0 & 0 & 0,0 & 1 & 0 & 0 & 1 & 0,3 & 0 & 0 & 0 & 0,0 & 1 & 2 \\
\hline Megascops choliba & 0 & 0 & 0 & 0,0 & 0 & 1 & 0 & 0,3 & 1 & 0 & 0 & 0 & 0,0 & 0 & 1 & 0 & 0,3 & 1 & 2 \\
\hline Gymnomystax mexicanus & 0 & 2 & 0 & 0,7 & 0 & 0 & 0 & 0,0 & 2 & 0 & 0 & 0 & 0,0 & 0 & 0 & 0 & 0,0 & & 2 \\
\hline Ramphastos vitellinus & 0 & 1 & 0 & 0,3 & 0 & 0 & 0 & 0,0 & 1 & 0 & 0 & 0 & 0,0 & 0 & 0 & 0 & 0,0 & & 1 \\
\hline Machaeropterus striolatus & 0 & 0 & 1 & 0,3 & 0 & 0 & 0 & 0,0 & 1 & 0 & 0 & 0 & 0,0 & 0 & 0 & 0 & 0,0 & & 1 \\
\hline Patagioenas cayennensis & 0 & 0 & 0 & 0,0 & 0 & 0 & 0 & 0,0 & & 0 & 1 & 0 & 0,3 & 0 & 0 & 0 & 0,0 & 1 & 1 \\
\hline Turdus ignobilis & 0 & 0 & 0 & 0,0 & 0 & 0 & 0 & 0,0 & & 1 & 0 & 0 & 0,3 & 0 & 0 & 0 & 0,0 & 1 & 1 \\
\hline Total & 32 & 55 & & & 31 & 8 & 3 & & 174 & 120 & 190 & 91 & & 143 & 76 & 76 & & 696 & 870 \\
\hline
\end{tabular}




\author{
Andrea Morales Rozo \\ Universidad de Los Llanos \\ Villavicencio, Colombia \\ amoralesrozo@unillanos.edu.co \\ https:/ / orcid.org/0000-0002-6920-8438

\section{Diego J. Lizcano} \\ The Nature Conservancy \\ Bogotá, Colombia \\ diego.lizcano@tnc.org \\ https:/ / orcid.org/0000-0002-9648-0576
}

\section{Sergio Montoya Arango}

Investigador independiente

sergioama123@gmail.com

https:/ / orcid.org/0000-0001-8734-7514

\author{
Álvaro Velásquez Suarez \\ Universidad de Los Llanos \\ Villavicencio, Colombia \\ alvaro.velasquez@unillanos.edu.co \\ https:/ / orcid.org/0000-0002-8126-4758
}

\section{Evelyn Álvarez Daza}

Universidad de Los Llanos

Villavicencio, Colombia

evelyn.alvarez@unillanos.edu.co

https:/ / orcid.org/0000-0001-6764-9523

\section{Orlando Acevedo-Charry}

Instituto de Investigación de Recursos Biológicos

Alexander von Humboldt

Villa de Leyva, Colombia

oacevedo@humboldt.org.co

https:/ / orcid.org/0000-0003-4964-8994
Heterópteros acuáticos y semiacuáticos asociados al bosque seco del Patía, suroeste de Colombia

Citación del artículo: Morales-Rozo, A., Lizcano, D., Montoya-Arango, S., Velásquez-Suarez, A., Álvarez-Daza, E. \& Acevedo-Charry, O. (2021). Diferencias en paisajes sonoros de sistemas silvopastoriles y potreros tradicionales del piedemonte llanero, Meta, Colombia. Biota Colombiana, 22(1), 74-95.

https:/ / doi.org/10.21068/c2021.v22n01a05

Recibido: 30 de mayo 2020

Aceptado: 9 de noviembre 2020 